Article

\title{
On an SEIR Epidemic Model with Vaccination of Newborns and Periodic Impulsive Vaccination with Eventual On-Line Adapted Vaccination Strategies to the Varying Levels of the Susceptible Subpopulation
}

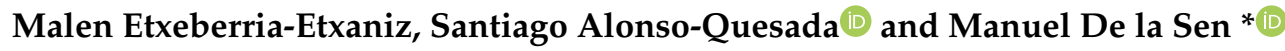 \\ Institute of Research and Development of Processes IIDP, Department of Electricity and Electronics, \\ University of the Basque Country, Campus of Leioa, 48940 Leioa, Spain; malen.etxeberria@gmail.com (M.E.-E.); \\ santiago.alonso@ehu.eus (S.A.-Q.) \\ * Correspondence: manuel.delasen@ehu.eus
}

Received: 26 October 2020; Accepted: 20 November 2020; Published: 23 November 2020

\begin{abstract}
This paper investigates a susceptible-exposed-infectious-recovered (SEIR) epidemic model with demography under two vaccination effort strategies. Firstly, the model is investigated under vaccination of newborns, which is fact in a direct action on the recruitment level of the model. Secondly, it is investigated under a periodic impulsive vaccination on the susceptible in the sense that the vaccination impulses are concentrated in practice in very short time intervals around a set of impulsive time instants subject to constant inter-vaccination periods. Both strategies can be adapted, if desired, to the time-varying levels of susceptible in the sense that the control efforts be increased as those susceptible levels increase. The model is discussed in terms of suitable properties like the positivity of the solutions, the existence and allocation of equilibrium points, and stability concerns related to the values of the basic reproduction number. It is proven that the basic reproduction number lies below unity, so that the disease-free equilibrium point is asymptotically stable for larger values of the disease transmission rates under vaccination controls compared to the case of absence of vaccination. It is also proven that the endemic equilibrium point is not reachable if the disease-free one is stable and that the disease-free equilibrium point is unstable if the reproduction number exceeds unity while the endemic equilibrium point is stable. Several numerical results are investigated for both vaccination rules with the option of adapting through ime the corresponding efforts to the levels of susceptibility. Such simulation examples are performed under parameterizations related to the current SARS-COVID 19 pandemic.
\end{abstract}

Keywords: SEIR epidemic model; vaccination of newborns; periodic impulsive vaccination; equilibrium points; periodic solutions; COVID-19 pandemic

\section{Introduction}

Epidemiology—from the Greek epi (about), demos (people), and logos (study)—-tries to study the distribution and determinant factors of diseases and applies this study to their control and prevention. It integrates procedures and techniques from disciplines such as the biomedical sciences and the social sciences [1-4]. One of the first works on the subject was proposed by Kermack and McKendrick in [2] where an SIR (susceptible-infectious-recovered) epidemic model with the host population being split into three categories depending on the status of the individuals with respect to the disease was proposed. Multiple studies and investigations have been carried out, thus developing new variants of this model, such as SEIR (susceptible-exposed-infectious-recovered) or SIS (susceptible-infectious-susceptible) [3,4]. The use of mathematical models, epidemiological models, constitutes an essential part of epidemiology, 
as it is an excellent tool for the analysis and prediction of the spread of diseases. They also make it possible to simulate the practical consequences of different intervention and control measures-such as vaccination or partial or total quarantine interventions-and, thus, to determine the most appropriate strategy to be invoked depending on the epidemiological situation. From the mathematical point of view, the simplest epidemiological models are of deterministic nature and they are formulated using systems of either ordinary differential or in difference equations, where the independent variable is time. The epidemic mathematical models have been applied to study real infectious disease and they can be adequate, both in their structure and in their parameterization, to the particular disease under study. Particular examples of such a model adequacy are, for instance, Bombay's epidemic pest of 1906 [5], the current COVID-19 pandemic [6-8], or measles and poliomyelitis [9,10]. In these two last cases, a periodic vaccination was proposed for a proportion of the susceptible based on an SIR epidemic model. Moreover, the strategy has been extended to impulsive periodic vaccination on more complex models, like for instance, SEIR models for cholera disease [11], each with their corresponding disease-related parameterizations [12].

The subpopulations under consideration usually change according to the model, but they always consist of groups or compartments into which the total population to be analyzed is divided. This work is only concerned with this type of model. However, there are different ways of formulating them, for example, considering the discrete time variable [13] instead of continuous; involving systems of equations in differences; or introducing random elements, so that the model has stochastic dynamics [14], which is a more realistic approach, but also more complicated to deal with. With regard to diseases, epidemiology deals with the modeling of infectious diseases-such as seasonal flu, chickenpox or the very recent COVID-19-even though epidemiological models are not limited only to this type of disease. However, it is in this case where development has been greatest in the last decades [1] since the disease spread is a crucial factor in them, and epidemiological models offer a very good tool to deal with it.

In [15-19], different types of vaccination strategies with eventual different application calendars and their achievable expected immunity are discussed. The fast and efficient tracking aspects of eventual infectious/susceptible infective contacts are examined in [20-25] in order to be able to achieve a prompt detection of new infective cases so as to try to avoid hospital collapse and a number of disease-related deaths. This concern has taken special relevance with the evolution of the current COVID-19 pandemic. The well-posedness of an epidemic model requires its fulfillment of basic properties like, for instance, the positivity of the solution under any non-negative initial conditions for any of the integrated subpopulations [26] as expected, since one deals with a biological problem and its boundedness and stability for all time [27-31], since the whole population in the habitat under study is bounded by nature. More recent studies on epidemic models have been developed in [32] where fractional calculus is used to amend more basic epidemic models. Some recent SEIR models being parameterized and adapted for the description of the evolution of the COVID-19 pandemic have been considered in [33-36] and some references therein. In [37,38], Shannon's information entropy is considered to study the transients in epidemic models focused on keeping the hospital availability of beds and hospital staff duties and other technical means below a certain admissible tolerance. See also some references therein. In [39], a very general true-mass SEIR epidemic model is studied and tested under mixed regular and impulsive vaccination controls while keeping the needed properties of positivity and stability. In $[40,41]$ and some references therein, stochastic epidemic models are described and analyzed subject to eventual altering noise on the nominal deterministic equilibrium steady-states. On the other hand, an SEIR epidemic normalized model under periodic impulsive vaccination is proposed and analyzed in [42]. The initial simpler version of the model in the paper is assumed to be subject to a recruitment parameter, which is coincident with the natural mortality rate and the disease is assumed to be mortality-free. The dynamics of the recovered subpopulation are not considered in the analysis by taking advantage of the fact that the total population remains constant over time due to the absence of disease mortality. Afterwards, the model is generalized by incorporating a disease 
mortality rate. Furthermore, Floquet's theory on stability of periodic solutions is involved in the proposed analysis without an explicit calculation of the Floquet's eigenvalues which conform some of the given conditions for the stability of the periodic oscillations. It is also proven mathematically in the paper that, in some cases, the local asymptotic stability ensures the global one, and that the periodic impulsive vaccination is slightly more efficient than the continuous traditional strategy.

In this paper, the application of both the newborns and impulsive vaccination strategies in the SEIR model are studied. We reconsider the problem of an SEIR model under an additional specific disease mortality rate under vaccination of newborns, which can be interpreted as an ad hoc modification of the recruitment in the susceptible subpopulation. Such a vaccination effort can be optionally accommodated to the current amounts of susceptible subpopulation. The basic objective is to study the stability of the equilibrium solutions of the system - both in the case of newborn vaccination and in the case of impulsive vaccination - for the proposed model, which helps to understand their long-term behavior. Concerning the newborn vaccination, it is demonstrated, both analytically and numerically, that the proposed vaccination strategy can lead to the eradication of an endemic disease. The disease-free and the endemic equilibrium points are both characterized explicitly, their local asymptotic stability properties are formally discussed based on the calculation of the next generation matrix and its eigenvalues, and the reproduction number is explicitly linked to the vaccination effort. Furthermore, the instability (respectively, the stability) of the disease-free equilibrium point is seen to coincide with the attainability and stability (respectively, the non-attainability) of the endemic one. The local asymptotic stability is seen to also be global by combining properties of non-negativity of the solution with the values of Poincare index for individual or groups of singular related to the discussion on the existence of limit cycles. Concretely, the local asymptotic stability is proven to also be global on the basis of the fact that the stable attainable attractor is the unique disease-free equilibrium point if reproduction number is less than unity (in which case the endemic equilibrium point is not attainable). Contrarily, if the disease-free equilibrium point is unstable, in the case of a reproduction number exceeding unity, then it is shown that it cannot be surrounded by a limit cycle in any of the phase planes; the only stable and attainable attractor being then the endemic equilibrium point. Another alternative vaccination strategy, which is also discussed in the paper under the same basic model, consists of a periodic impulsive vaccination. Again, the vaccination effort can be accommodated to the current stocks of susceptible subpopulation. The stability properties of the periodic steady-state are performed via Floquet's theory, with an explicit calculation of the eigenvalues of the monodromy matrix which are the Floquet multipliers. This method allows us to establish that, under a sufficiently small transmission rate, the local asymptotic stability is guaranteed according to a formula which makes explicit a trade-off between the vaccination interval between consecutive vaccination pulses and the susceptible population fraction to be vaccinated by a defining a reproduction-like number. To perform the above summarized study, this work is split into five sections which are organized as follows. Section 2 aims at establishing and discussing the SEIR model under eventual vaccination of newborns. The general formulation of the proposed SEIR model is given. Later on, the mathematical analysis of the model properties is discussed in detail in terms of local and global asymptotic stability concerns of both the disease-free and endemic equilibrium points, which are explicitly calculated as dependent of the vaccination of newborns levels. It is proven that the endemic equilibrium point is not reachable when the disease-free one is locally asymptotically stable, which happens as the basic reproduction number is less than unity. It is also proven that when the basic reproduction number exceeds unity then the endemic equilibrium point is reachable and locally asymptotically stable while the disease-free one is unstable. The reproduction number is improved with the levels of vaccination, which means that the reproduction number is kept below unity for wider ranges of potential values of the transmission rate as the vaccination effort increases. It is also proven that the local asymptotic stability of the equilibrium points also imply both respective global asymptotic stability properties. In the formulation of Section 2, the absence of vaccination of newborns might be understood as a particular case of the corresponding vaccination effort. On the other hand, 
in Section 3, an impulsive periodic vaccination strategy is proposed and studied, and the resulting model properties are formulated for the delay-free case. In this case, it is necessary to work with a resulting dynamic system of periodic coefficients, and, for this purpose, the Floquet theory is used to formulate the stability properties. Some numerical results are displayed and discussed in Section 4 for the COVID-19 pandemic, which are then performed to visualize the solutions for the two studied vaccination strategies. First, an endemic disease is simulated using the SEIR model, and then the mentioned vaccination strategies which are applied with the aim of eradicating the disease or, at least, softening its impact. To complete the section, two new control methods are designed by modifying the strategies presented by adapting them to the recruitment or susceptible levels in the sense that the control efforts are reinforced through time as such levels become larger. In this way, an attempt is made to propose new strategies that achieve eradication of the disease with less vaccination coverage, which is usually interesting in the context of the logistics of vaccination campaigns. The code for the programs developed for the simulations in this chapter has not been included in this report but can be found in the malenetxeberria/TFG-IngElec repository on GitHub. Finally, conclusions end the paper.

Compared to some related previous work, note that, in [10], the pulse vaccination strategy is programmed on an SIR epidemic model while in the current paper, the impulsive periodic vaccination is proposed for an SEIR epidemic model. There are recent studies for COVID-19 concerns, see, for instance [20-25,36]. However, those studies do not consider the potential application of vaccination which really does not yet exist in good condition for its mass application. Furthermore, most of the studies refer to short-term evaluations and prediction of the disease behavior. In the current work, two vaccination strategies are proposed for parameterizations associated to the COVID-19 pandemic. In particular, the second proposed strategy of periodic impulsive vaccination may be appropriate to deal with successive waves of serious incidence and later decrease of the infection. Furthermore, the vaccine administration strategies can be adapted to the levels of susceptible individuals which can facilitate the administration calendar.

\section{SEIR Epidemic Model with Eventual Vaccination of Newborns}

\subsection{The Epidemic Model}

An SEIR epidemic model is proposed where the host population is split into four categories, namely:

- Susceptible: individuals who have not contracted the disease but are at risk for it, since they do not have the necessary antibodies to cope with it.

- Exposed: this is the group of individuals who are already infected, but who are not yet infectious, so they cannot transmit the infection. This state lasts for a limited period of time that varies according to the disease, and is known as the latent period [1] (Chap. Two).

- Infectious: those individuals that are infected and can transmit the disease.

- Recovered: individuals who have managed to defeat the disease. The body generates immunity as an adaptive response to the infectious agent [15], so that a recovered individual is no longer susceptible.

It is considered that the population is homogeneously mixed, that its spatial distribution is uniform, and that all susceptible individuals have the same probability of being infected, which simplifies the problem considerably. The mathematical representation of the SEIR model considered in this case [3] is given by the system of differential equations:

$$
\begin{gathered}
\dot{S}=(1-q) A-(\beta I+\mu) S \\
\dot{E}=\beta S I-(\mu+\sigma) E \\
\dot{I}=\sigma E-(\mu+\gamma+\alpha) I \\
\dot{R}=q A+\gamma I+\mu R
\end{gathered}
$$


subject to an initial condition given by: $S(0) \geq 0, E(0) \geq 0, I(0) \geq 0$ and $R(0) \geq 0$. The variables $S(t), E(t)$, $I(t)$, and $R(t)$ indicate the proportion of susceptible, exposed, infectious, and recovered individuals of the subpopulations, with respect to the total population, found in each compartment at a specific time. The sum of the four subpopulations at the initial time instant is assumed to be unity, in general, so that the subpopulations of the model are fractions of the total initial population. However, this is not a real constraint since the initial conditions can be also fixed to the real values of the various subpopulations. The parameters are constant with positive values related to either demographic characteristics or to the infection type.

The symbols used for each variable or parameter are as follows:

S: Proportion of susceptible individuals

E: Proportion of exposed individuals

I: Proportion of infectious individuals

$R$ : Proportion of recovered individuals

$A$ : Proportion of new individuals per unit of time

$\beta$ : Transmission rate

$\mu$ : Natural mortality rate

$\sigma$ : Inverse of the latent period

$\gamma$ : Recovery rate

$\alpha$ : Mortality rate caused by infection

$q$ : Fraction of vaccinated newborns

Note the following facts on the above model:

- New individuals enter the susceptible compartment per unit of time, either by birth or by immigration. $\beta S I$ indicates the proportion of infections [4], that is, the proportion of individuals who are no longer susceptible and who become exposed; and finally, $\mu \mathrm{S}$ is the proportion of individuals who die from causes not related to the infection, also per unit of time.

- The infected susceptible $\beta$ SI pass into the exposed compartment and are removed from it once the latent period has passed, that is, $\sigma E$ individuals are removed per unit of time. Naturally, the fraction $\mu \mathrm{E}$ of those exposed individuals who die from causes unrelated to infection must also be considered.

- At the end of the latent period, the exposed $\sigma \mathrm{E}$ become infectious. Moreover, $\gamma \mathrm{I}$ is the proportion of individuals who overcome the disease and who, therefore, pass to the recovered compartment; instead, $\alpha \mathrm{I}$ represents the individuals who die from the infection, and who are removed from the population.

- $\gamma \mathrm{I}$ individuals enter the recovered compartment per unit of time, and $\mu \mathrm{R}$ are withdrawn due to mortality from causes not related to the infection.

- The fraction of vaccinated newborns is directly added to the recovered compartment and withdrawn from the susceptible one.

In this way, the scheme of the SEIR model is represented in Figure 1.

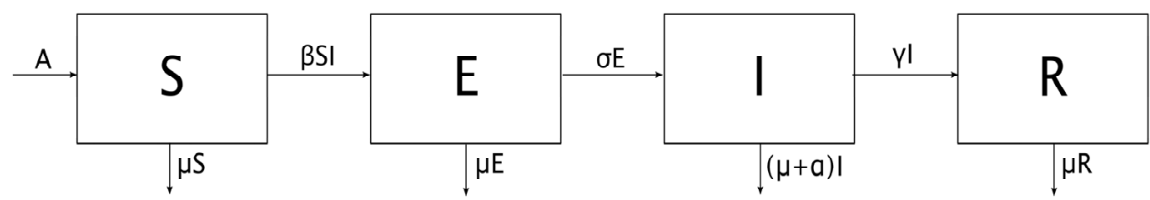

Figure 1. Graphic representation of the susceptible-exposed-infectious-recovered (SEIR) model. 


\subsection{Non-Negativity of the Solution}

The solutions of Equation (1) are non-negative for all time for any given non-negative initial condition. This is easily proven by calculating, in a closed analytic form, the unique solution of Equation (1) for any set of non-negative initial conditions. This property is needed for well-posedness of the epidemic model Equation (1). In parallel, the fact that the total population is proven to be bounded for all time guarantees also the boundedness for all time of all the subpopulations under any given arbitrary finite non-negative initial conditions.

Theorem 1. The following properties hold for any $q \in[0,1]$ :

(i) The solution trajectory of the epidemic model (1) is non-negative for all time for any given non-negative initial conditions.

(ii) The solution trajectory of Equation (1) is bounded for all time for any given finite non-negative initial conditions and $\lim \sup (t) \leq A \min (1 / \alpha, 1 / \mu)$. As a result, the epidemic model is globally stable for any given arbitrary finite non-negative initial conditions.

Proof. The solution to (1) is

$$
\begin{gathered}
S(t)=e^{-\int_{0}^{t}(\beta I(\tau)+\mu) d \tau} S(0)+(1-q) A \int_{0}^{t} e^{-\int_{\tau}^{t}(\beta I(s)+\mu) d s} d \tau \\
E(t)=e^{-(\mu+\sigma) t} E(0)+\beta \int_{0}^{t} e^{-(\mu+\sigma)(t-\tau)} S(\tau) I(\tau) d \tau \\
=e^{-(\mu+\sigma) t} E(0)+\beta \int_{0}^{t} e^{-(\mu+\sigma)(t-\tau)} S(\tau)\left[e^{-(\mu+\gamma+\alpha) \tau} I(0)+\sigma \int_{0}^{\tau} e^{-(\mu+\gamma+\alpha)(\tau-s)} E(s) d s\right] d \tau \\
I(t)=e^{-(\mu+\gamma+\alpha) t} I(0)+\sigma \int_{0}^{t} e^{-(\mu+\gamma+\alpha)(t-\tau)} E(\tau) d \tau \\
R(t)=e^{-\mu t} R(0)+\int_{0}^{t} e^{-\mu(t-\tau)}(\gamma I(\tau)+q A) d \tau
\end{gathered}
$$

It is obvious that $S(t) \geq 0$ for all time if $S(0) \geq 0$ from the above first equation. Then, $E(t) \geq 0$ for all time from the second equality of the above second equation if $S(0) \geq 0, E(0) \geq 0$, and $I(0) \geq 0$ since $S(t) \geq 0$ for all time. Subsequently, $I(t) \geq 0$ for all time from the above third equation if $I(0) \geq 0$, since $E(t) \geq 0$ for all time. Finally, $R(t) \geq 0$ for all time from the above fourth equation if $R(0) \geq 0$, since $I(t) \geq 0$ for all time. Property (i) has been proved. By summing-up the four subpopulations in Equation (1), one gets that the total population $N(t)=S(t)+E(t)+I(t)+R(t)$ satisfies the differential Equation (3)

$$
\dot{N}(t)=\dot{S}(t)+\dot{E}(t)+\dot{I}(t)+\dot{R}(t)=-\mu N(t)+A-\alpha I(t)
$$

whose solution is

$$
N(t)=e^{-\mu t} N(0)+\int_{0}^{t} e^{-\mu(t-\tau)}(A-\alpha I(\tau)) d \tau \leq e^{-\mu t} N(0)+\frac{A}{\mu} \leq N(0)+\frac{A}{\mu}<+\infty
$$

so that the total population is bounded for all time. From Property (i), since all the subpopulations are non-negative for all time, it follows that they are also bounded for all time and that $N(t) \geq 0$ for all time. In addition, $\limsup _{t \rightarrow \infty} I(t) \leq \limsup _{t \rightarrow \infty} N(t) \leq A / \mu$ from the above equation. Furthermore $\limsup _{t \rightarrow \infty} I(t) \leq A / \alpha$ (otherwise, if $\limsup _{t \rightarrow \infty} I(t)>A / \alpha$ then $\lim _{t \rightarrow \infty} N(t)=-\infty$, a contradiction). Therefore, $\limsup _{t \rightarrow \infty} I(t) \leq A \min (1 / \alpha, 1 / \mu)$. 


\subsection{Equilibrium Points}

There are two equilibrium points, that is, the disease-free one, which is obtained from (1) by equalizing to zero all the time-derivatives of Equation (1) and equalizing the infectious to zero. This leads to

$$
E Q^{0}(q)=\left(S^{0}(q), E^{0}, I^{0}, R^{0}(q)\right)=((1-q) A / \mu, 0,0, q A / \mu)
$$

On the other hand, the endemic equilibrium point is obtained from Equation (1) by equalizing again to zero all the time-derivatives while keeping a non-zero infectious subpopulation which yields:

$$
E Q^{*}(q)=\left(S^{*}(q), E^{*}(q), I^{*}(q), R^{*}(q)\right)
$$

where:

$$
\begin{gathered}
S^{*}(q)=\frac{(1-q) A}{\beta I^{*}+\mu} \\
E^{*}(q)=\frac{\mu+\gamma+\alpha}{\sigma} I^{*} \\
I^{*}(q)=\frac{(1-q) A \sigma}{(\mu+\sigma)(\mu+\gamma+\alpha)}-\frac{\mu}{\beta} \\
R^{*}(q)=\frac{\gamma I^{*}+q A}{\mu}
\end{gathered}
$$

Recombining the above equations yields:

$$
\begin{gathered}
S^{*}(q)=\frac{(\mu+\sigma)(\mu+\gamma+\alpha)}{\beta \sigma} \\
E^{*}(q)=\frac{(1-q) A}{\mu+\sigma}-\frac{\mu(\mu+\gamma+\alpha)}{\beta \sigma} \\
I^{*}(q)=\frac{(1-q) \sigma A}{(\mu+\sigma)(\mu+\gamma+\alpha)}-\frac{\mu}{\beta} \\
R^{*}(q)=\frac{A}{\mu}\left(\frac{(1-q) \gamma \sigma}{(\mu+\sigma)(\mu+\gamma+\alpha)}+q\right)-\frac{\gamma}{\beta}
\end{gathered}
$$

From Equation (5) into Equation (3) equalized to zero yields the total endemic equilibrium population:

$$
N^{*}=\frac{A-\alpha I^{*}}{\mu}=\frac{A}{\mu}\left(1-\frac{(1-q) \alpha \sigma}{(\mu+\sigma)(\mu+\gamma+\alpha)}\right)+\frac{\alpha}{\beta}
$$

Note that all the subpopulations of Equation (5), see also Equation (6), have to be non-negative for the endemic equilibrium point to exist in the first orthant of the state-space so that it be reachable. Otherwise, Theorem 1 would be violated. Thus, we have the subsequent result:

Proposition 1. The endemic equilibrium point is reachable if and only if the transmission rate is large enough to be non less than a critical value according to:

$$
\beta \geq \beta_{c}(q)=\frac{\mu(\mu+\sigma)(\mu+\gamma+\alpha)}{A \sigma(1-q)}
$$

for any $q \in[0,1)$. If the transmission rate equalizes its critical value, then the endemic equilibrium point is coincident with the disease-free one. 


\subsection{Basic Reproduction Number and Stability of the Equilibrium Points}

One of the most important parameters in epidemiology is the basic reproduction number $R_{0}$, which is defined as the average number of secondary cases caused by the entry of an infectious individual into a totally susceptible population [1] (Chap. 2). There are several techniques for calculating the basic reproduction number, and in this work, the next generation method will be shown [16], where $R_{0}$ is the spectral radius of the next generation matrix. So, to build this matrix, first, one defines the transmission and transition function matrices $\mathbf{F}$ and $\mathbf{- V}$ defined from the second and third equations of (1) by:

$$
\mathbf{F}=\left(\begin{array}{c}
\beta S I \\
0
\end{array}\right) ; \mathbf{V}=\left(\begin{array}{c}
(\mu+\sigma) E \\
-\sigma E+(\mu+\gamma+\alpha) I
\end{array}\right)
$$

which are evaluated at the disease-free equilibrium point to yield the matrix of dynamics of the linearized system around the disease-free equilibrium point, that is, the Jacobian matrix of the infective subsystem $(E, I)$ at such a point,

$$
\hat{\mathbf{J}}_{E Q^{0}}=\mathbf{F}-\mathbf{V}=\left(\frac{\partial F}{\partial x}\right)_{E Q^{0}}-\left(\frac{\partial V}{\partial x}\right)_{E Q^{0}}=-\mathbf{V}\left(\mathbf{I}_{2}-\mathbf{V}^{-1} \mathbf{F}\right)
$$

Ref. [38], where $\mathbf{I}_{2}$ is the identity matrix of second order. Since $\left.-\mathbf{V}=\hat{\mathbf{J}}_{E Q^{0}}\right]_{\beta=0}$ is a stability matrix since the linearized system around the disease-free equilibrium point is globally asymptotically stable in the absence of disease, that is, if the transmission rate is zero, one follows from Equation (9) that $\hat{\mathbf{J}}_{E Q^{0}}$ is a stability matrix if and only if the spectral radius of $\mathbf{V}^{-1} \mathbf{F}$ is less than one or, equivalently, that of

$$
\mathbf{G}=\mathbf{F V}^{-1}=\left[\begin{array}{cc}
\frac{A \sigma(1-q) \beta}{\mu(\mu+\sigma)(\mu+\gamma+\alpha)} & \frac{A(1-q) \beta}{\mu(\mu+\gamma+\alpha)} \\
0 & 0
\end{array}\right]
$$

that is

$$
R_{0}(q)=\frac{A \sigma(1-q) \beta}{\mu(\mu+\sigma)(\mu+\gamma+\alpha)}
$$

referred to as the basic reproduction number, is less than one, that is, if and only if $\beta<\beta_{c}(q)$ and this Jacobian matrix is critically stable for $\beta=\beta_{c}(q)$ and unstable for $\beta>\beta_{c}(q)$. Comparing this result with Proposition 1, and since the eigenvalues of the Jacobian matrix Equation (9) of the infective pair $(E, I)$ are continuous functions of the transmission rate, yields the result that the disease-free equilibrium point is asymptotically stable if and only if the endemic one is unreachable, which happens for a sufficiently small transmission rate as follows:

Theorem 2. The disease-free equilibrium point is the only reachable equilibrium point and it is globally asymptotically stable if and only if the transmission rate is small enough to satisfy $\beta \in\left[0, \beta_{c}(q)\right)$. It is coincident with the endemic equilibrium point and critically stable if $\beta=\beta_{c}(q)$ and unstable if $\beta>\beta_{c}(q)$.

Proof. One has from the above discussion that for sufficiently close to the disease-free equilibrium point, $E(t) \rightarrow 0$ and $I(t) \rightarrow 0$ as $t \rightarrow \infty$ if $\beta<\beta_{c}(q)$. On the other hand, note from Proposition 1 that, if $\beta<\beta_{c}(q)$, then the exposed subpopulation is negative which contradicts Theorem 1(i). Therefore, the endemic equilibrium is not attainable if the reproduction number is less than one so that the only global stable attractor is the disease-free equilibrium point. On the other hand, all the subpopulations are bounded for all time and any $\beta \geq 0$ from Theorem 1. So that: (a) either $S(t) \rightarrow(1-q) A / \mu$, $R(t) \rightarrow q A / \mu$ as $t \rightarrow \infty$ and the result is already proven; (b) at least one of $S(t)$ and $R(t)$ is unbounded as time increases, which is impossible since this unboundedness would contradict Theorem 1 ; (c) at 
least one of $S(t)$ and $R(t)$ exhibits asymptotic oscillations. However, then from the solutions in the proof of Theorem 1 and since $I(t) \geq 0$ for all and $t \geq 0$ as $t \rightarrow \infty$ :

$$
\begin{gathered}
\lim _{t \rightarrow \infty}\left(S(t)-(1-q) A \int_{0}^{t} e^{-\int_{\tau}^{t}(\beta I(s)+\mu) d s} d \tau\right)=0 ; \lim _{t \rightarrow \infty}\left(R(t)-\int_{0}^{t} e^{-\mu(t-\tau)}(\gamma I(\tau)+q A) d \tau\right)=0 \\
\lim _{t \rightarrow \infty} \int_{0}^{t} e^{-\int_{\tau}^{t}(\beta I(\sigma)+\mu) d \sigma} d \tau=C_{1}>0 ; \lim _{t \rightarrow \infty} \int_{0}^{t} e^{-\mu(t-\tau)}(\gamma I(\tau)+q A) d \tau=C_{2} \geq 0
\end{gathered}
$$

therefore, $S(t)$ and $R(t)$ cannot oscillate asymptotically so that they converge to finite non-negative limits. Since the disease-free equilibrium point is unique, then $S(t) \rightarrow S^{0}(q)=(1-q) A / \mu$ and $R(t) \rightarrow R^{0}(q)=q A / \mu$ as $t \rightarrow \infty$, and then the disease-free equilibrium point is (not only locally but also globally) asymptotically stable if and only if $\beta \in\left[0, \beta_{c}(q)\right)$. If $\beta>\beta_{c}(q)$, then the spectral radius of the subsystem of the infective variables $(E, I)$ exceeds unity so that the corresponding sub-matrix of the Jacobian matrix is unstable and then the whole Jacobian matrix at the disease-free equilibrium point is unstable. If $\beta=\beta_{c}(q)$, then some eigenvalue of the Jacobian matrix of the disease-free equilibrium point is at the stability boundary and the linearized system is critically stable.

Alternative Proof. Consider the whole Jacobian matrix at the disease-free equilibrium point:

$$
\mathbf{J}_{E Q^{0}}(q)=\left(\frac{\partial x^{T}}{\partial x}\right)_{E Q^{0}}=\left[\begin{array}{cccc}
-\mu & 0 & -\frac{\beta(1-q) A}{\mu} & 0 \\
0 & -(\mu+\sigma) & \frac{\beta(1-q) A}{\mu} & 0 \\
0 & \sigma & -(\mu+\gamma+\alpha) & 0 \\
0 & 0 & \gamma & -\mu
\end{array}\right]
$$

where $x^{T}=(S, E, I, R), S^{0}(q)=(1-q) A / \mu$, and $\left(E^{0}, I^{0}, R^{0}\right)=(0,0,0)$. The eigenvalues of Equation (12) are

$$
\begin{gathered}
\lambda_{1,2}=-\mu<0 \\
\lambda_{3,4}=\frac{1}{2}\left[-(\alpha+\sigma+\gamma+2 \mu) \pm \sqrt{(\alpha-\sigma)^{2}+\gamma(\gamma+2(\alpha-\sigma))+\frac{4 \beta(1-q) \sigma A}{\mu}}\right] \\
=\frac{1}{2}\left[-(\alpha+\sigma+\gamma+2 \mu) \pm \sqrt{(\gamma+\alpha-\sigma)^{2}+\frac{4 \beta(1-q) \sigma A}{\mu}}\right]
\end{gathered}
$$

Note that $\lambda_{3}$ and $\lambda_{4}\left(<\lambda_{3}\right)$ are real eigenvalues of the above Jacobian matrix. Then, the Jacobian matrix at the disease-free equilibrium point is a stability matrix if and only if $\lambda_{3}<0$, that is, if and only if

$$
(\alpha+\sigma+\gamma+2 \mu)^{2}>(\gamma+\alpha-\sigma)^{2}+\frac{4 \beta(1-q) \sigma A}{\mu}
$$

which holds if and only if the transmission rate is small enough to satisfy $\beta<\beta_{c}(q)$ or, equivalently, if the basic reproduction number Equation (11) is less than one, equivalently $R_{0}(q)<1$. If the inequality Equation (14), equivalently $R_{0}(q)<1$, becomes an equality, still equivalent to $R_{0}(q)=1$ in (11), then $\lambda_{3}=0$, that is, one eigenvalue of Equation (12) is critically stable and the disease-free equilibrium point is then critically stable. If the inequality in Equation (14) is reversed $\left(R_{0}(q)>1\right)$ then the disease-free equilibrium point is unstable.

Finally, note that the local asymptotic stability of the disease-free equilibrium point for $R_{0}(q)<1$ is also global since:

(a) it is the unique attainable equilibrium point since the endemic one is not reachable from Proposition 1. 
(b) the first right-hand-side term of Equation (2) tends asymptotically to zero as time tends to infinity so that, irrespective of the initial conditions, either the following limit exists:

$$
\lim _{t \rightarrow \infty}\left(S(t)-(1-q) A \int_{0}^{t} e^{-\int_{\tau}^{t}(\beta I(s)+\mu) d s} d \tau\right)=0
$$

which implies that the susceptible subpopulation tends to a finite and non-negative limit (from Theorem 1) as time tends to infinity, which is the disease-free equilibrium susceptible subpopulation. In this case, the (non-negative) integrand of the second term of the above limit tends asymptotically to zero; or

(c) it converges to a periodic solution of some period $T>0$, that is, $S(n k T+\tau) \rightarrow S^{*}(\rho, q)$ for some continuous $\rho:[0, T) \rightarrow \mathbf{R}_{0+}$ as $n\left(\in \mathbf{Z}_{0+}\right) \rightarrow \infty ; \forall k \in \mathbf{Z}_{+}$. $\square$

If the image of $\rho:[0, T) \rightarrow \mathbf{R}_{0+}$ is a real constant, then the convergence is to a point for all $\rho \in[0, T)$ which is the susceptible component of the disease-free equilibrium point which is already discussed. If $\rho:[0, T) \rightarrow \mathbf{R}_{0+}$ is not constant, then the susceptible subpopulation converges to a periodic solution. However, note that the second right-hand-side term of the first equation of (2) has a non-negative integrand so that either it converges to zero in order for $S(t)$ to be bounded for all time or $S(t)$ is asymptotically unbounded which violates Theorem 1 . As a result, $S(t)$ cannot exhibit an asymptotic oscillation while it converges to the disease-free susceptible equilibrium point as time tends to infinity.

Now, consider the two-dimensional phase plane $(\dot{E}, \dot{I})=(f(E, I), g(E, I))$ at the disease-free susceptible equilibrium point $S^{*}(q)$. Since the reproduction number is less than one, the equilibrium point cannot be a saddle point, then its Poincaré index is +1 and no stable limit cycle can surround it. Thus, the disease-free equilibrium point, which is a local asymptotic attractor and the unique stable global attractor, is also the unique global asymptotic attractor if the reproduction number is less than one.

Remark 1. Note that, for any $q \in[0,1)$, the basic reproduction number in the case of vaccination of newborns becomes reduced to $R_{0}(q)=(1-q) R_{0}(0)$ compared to the case of absence of vaccination (whose corresponding reproduction number is $R_{0}(0)$ ), and then the disease-free equilibrium point is globally asymptotically stable if and only if $R_{0}(q)<1$. This implies that, under a vaccination of newborns effort, the global asymptotic stability of the disease-free equilibrium point together with the non-reachability of the endemic one are achieved if and only if

$$
R_{0}(0) \in[0,1 /(1-q))
$$

Remark 2. An equivalent interpretation is that the stability of the disease-free equilibrium point and the parallel property of non-reachability of the endemic one are achievable for a larger range of transmission rate related to the absence of vaccination of newborns since the critical transmission rate satisfies $\beta_{c}(q)>\beta_{c}(0)$ if $q \in[0,1)$ (Proposition 1).

Remark 3. As a result of the above considerations, one can argue that vaccination of newborns effort is useful in achieving the asymptotic eradication of the disease since the range of transmission rate values being compatible with the stability of the disease-free equilibrium increases related to its parallel range of values in the absence of vaccination.

The subsequent result, whose proofs follow by direct calculation, relies on alternative equivalent expressions to Equation (6) on the components of the endemic equilibrium point depending on the reproduction number. 
Proposition 2. The components of the endemic equilibrium point may be rewritten, equivalently to (6), as follows:

$$
\begin{gathered}
S^{*}(q)=\frac{(1-q) A}{\mu R_{0}(q)} ; \quad E^{*}(q)=\frac{(1-q) A}{\mu+\sigma}\left(1-\frac{1}{R_{0}(q)}\right) \\
I^{*}(q)=\frac{\mu}{\beta}\left(R_{0}(q)-1\right) ; \quad R^{*}(q)=\frac{q A}{\mu}+\frac{\gamma}{\beta}\left(R_{0}(q)-1\right)
\end{gathered}
$$

It is reachable if and only if $R_{0}(q) \geq 1$ and it is coincident with the disease-free equilibrium point if and only if $R_{0}(q)=1$.

Theorem 3. The endemic equilibrium point is globally asymptotically stable for any $q \in[0,1]$ if and only if the transmission rate satisfies $\beta \in\left(\beta_{c}(q), \infty\right)$, equivalently, if and only if the basic reproduction number exceeds unity.

Proof. The Jacobian matrix at the endemic equilibrium point is:

$$
\mathbf{J}_{E Q^{*}}(q)=\left(\frac{\partial x^{T}}{\partial x}\right)_{E Q^{*}}=\left[\begin{array}{cccc}
-\mu R_{0}(q) & 0 & -\frac{(\mu+\sigma)(\mu+\gamma+\alpha)}{\sigma} & 0 \\
\mu\left(R_{0}(q)-1\right) & -(\mu+\sigma) & \frac{(\mu+\sigma)(\mu+\gamma+\alpha)}{\sigma} & 0 \\
0 & \sigma & -(\mu+\gamma+\alpha) & 0 \\
0 & 0 & \gamma & -\mu
\end{array}\right]
$$

One of the eigenvalues of (16) is $\lambda_{1}=-\mu$. Instead of calculating the other three eigenvalues of Equation (16), which is a difficult task, the Routh-Hurwitz stability criterion $p(\lambda)=\sum_{i=0}^{3} a_{3-i} \lambda^{i}=0$ is applied to the characteristic equation of the third order remaining submatrix [31], where

$$
\begin{gathered}
a_{0}=1 ; a_{1}=\mu\left(2+R_{0}(q)\right)+\gamma+\alpha+\sigma ; a_{2}=\mu R_{0}(q)(2 \mu+\gamma+\alpha+\sigma) ; \\
a_{3}=\mu\left(R_{0}(q)-1\right)(\mu+\gamma+\alpha)(\mu+\sigma) .
\end{gathered}
$$

The Routh-Hurwitz criterion is applied by first calculating the following determinants:

$$
\begin{gathered}
\Delta_{0}=1 ; \Delta_{1}=a_{1}=\mu\left(2+R_{0}(q)\right)+\gamma+\alpha+\sigma ; \Delta_{2}=\left|\begin{array}{cc}
a_{1} & a_{0} \\
a_{3} & a_{2}
\end{array}\right|=a_{1} a_{2}-a_{3} ; \\
\Delta_{3}=\left|\begin{array}{ccc}
a_{1} & a_{0} & 0 \\
a_{3} & a_{2} & a_{1} \\
0 & 0 & a_{3}
\end{array}\right|=a_{3} \Delta_{2} .
\end{gathered}
$$

The number of characteristic roots of $p(\lambda)=0$ with positive real part is the number of sign changes in the set $\left\{\Delta_{0}, \Delta_{1}, \Delta_{2} / \Delta_{1}, \Delta_{3} / \Delta_{2}\right\}$. Since $\Delta_{0}>0$ and $\Delta_{1}>0$, then there is no characteristic root with positive real part if and only if $\Delta_{2} / \Delta_{1}>0, \Delta_{3} / \Delta_{2}>0$ [28]. Note that $\Delta_{3} / \Delta_{2}>0$ if and only if $R_{0}(q)>1$, and $\Delta_{2} / \Delta_{1}>0$ if and only if

$$
a_{1} a_{2}-a_{3}=\mu\left[\mu\left(2+R_{0}(q)\right) R_{0}(q)(2 \mu+\gamma+\alpha+\sigma)+(\gamma+\alpha+\sigma)\left(R_{0}(q)(\mu+\gamma+\alpha)+\mu+\sigma\right)\right]>0
$$

which always holds. Then, (16) is a stability matrix if and only if $R_{0}(q)>1$. Thus, the endemic equilibrium point is locally asymptotically stable if and only if $R_{0}(q)>1$. It is now proven that such a stability property is global by using contradiction arguments. Assume that the solution is oscillatory periodic with some arbitrary oscillation period $T>0$. The period is supposed arbitrary since it is being rebutted by contradiction arguments in the following that some such oscillation period exits so that no limit oscillation exists as a result. Define $\hat{y}(\delta)=\lim _{n \rightarrow \infty} y(n T+\tau)$ and $\hat{y}_{M}(\delta)=\lim _{n \rightarrow \infty} \max _{\tau \in[0, \delta]} y(n T+\tau)$ for the four subpopulations $y=S, E, I, R$. Note that all such claimed maximum values are positive real 
numbers. From Equation (2) in the proof of Theorem 1, one gets for $t=n T+\delta, n \in \mathbf{Z}_{0+}=\{z \in \mathbf{Z}: z \geq 0\}$, and $\delta \in[0, T)$ that the limits below exist for any such a $\delta \in[0, T)$ :

$$
\begin{gathered}
\hat{S}(\delta)=\lim _{n \rightarrow \infty} S(n T+\delta)=(1-q) A \int_{0}^{\delta} e^{-\int_{\tau}^{\delta}(\beta I(s)+\mu) d s} d \tau \leq(1-q) A \delta ; \\
\hat{E}(\delta)=\lim _{n \rightarrow \infty} E(n T+\delta) \leq \beta \delta \hat{S}_{M}(\delta) \hat{I}_{M}(\delta) ; \hat{I}(\delta)=\lim _{n \rightarrow \infty} I(n T+\delta) \leq \sigma \delta \hat{E}_{M}(\delta) ; \\
\hat{R}(\delta)=\lim _{n \rightarrow \infty} R(n T+\delta) \leq \delta\left(\gamma \hat{I}_{M}(\delta)+q A\right)
\end{gathered}
$$

after using the mean value theorem for integrals since all the involved integrals in Equation (2) are continuous functions of time. Note that $\hat{y}:[0, T) \rightarrow \mathbf{R}_{0+}$ will define the periodic limit oscillation of the subpopulation $z$ in the event that such an oscillation exists. By recombining the three first above equations, one gets for any given $q \in(0,1]$, any $\delta \in(0, T]$, and any supposed arbitrary oscillation period $T>0$ that, since $\hat{E}(\tau)=\hat{E}_{M}(\delta)$ for some $\tau \in(0, \delta]$ and any $\delta \in(0, T]$ :

$$
\hat{E}(\tau) \leq \beta A \sigma(1-q) \delta^{3} \hat{E}_{M}(\delta) \Leftrightarrow 1 \leq \beta A \sigma(1-q) \delta^{3}
$$

which is violated if

$$
\delta \in\left(0, \min \left(T, \frac{1}{\sqrt[3]{\beta A \sigma(1-q)}}\right)\right)
$$

Hence, a contradiction follows such that the exposed subpopulation does not have a limit oscillation of any potential oscillation period, since a chaotic behavior cannot exist either since the model has no small time-varying parameter. Similarly, one gets a similar conclusion on no existence of limit oscillations of any period of the infectious subpopulation since the oscillation condition is violated for a range of sufficiently small inter-period time instants for any arbitrary claimed oscillation period according to:

$$
\hat{I}_{M}(\delta) \leq \beta \sigma(1-q) A \delta^{3} \hat{I}_{M}(\delta)
$$

and similar conclusions can be deduced for the susceptible and the recovered individuals. Therefore, no limit cyclic exists surrounding the reachable endemic equilibrium point when the disease-free equilibrium point is unstable, that is, if the basic reproduction number exceeds unity. Furthermore, all solutions are bounded from Theorem 1. As a result, the endemic equilibrium point is globally asymptotically stable if the basic reproduction number exceeds unity, equivalently, if and only if $\beta \in\left(\beta_{c}(q), \infty\right)$.

Remark 4. It can be of interest to give some considerations which are based on known general results of the qualitative theory of differential equations which indicate that there is a unique global asymptotically stable attractor which is always one of the equilibrium points [43-45]. The global attractor is the disease-free equilibrium if the reproduction number is less than or equal to one and the endemic equilibrium point if such a parameter exceeds unity. In this way, there are no stable oscillations surrounding neither one of the equilibrium points nor jointly both of them. If the reproduction number is unity, then the asymptotic behavior is similar to that arising if it is less than unity so that the disease-free equilibrium point is a global asymptotically stable attractor. Thus, the constraints implying local asymptotic stability of any of the equilibrium points lead to their global asymptotic stability. Those considerations are deduced from the subsequent linked arguments:

(a) If $R_{0}(q)<1$, then the disease-free equilibrium point is locally asymptotically stable and the endemic one is not reachable. A limit cycle surrounding it in any phase plane of two subpopulations, if any, would be unstable from the combined topology of equilibrium points and limit cycles. Therefore, it would be irrelevant for stability considerations. As a result, the disease-free equilibrium point is globally asymptotically stable (Theorem 2).

(b) If $R_{0}(q)>1$, then the endemic equilibrium point is locally asymptotically stable and the disease-free equilibrium point is unstable. A limit cycle surrounding it leaving away the disease-free one, if any, would be unstable for stability considerations. No limit cycles can exist surrounding both equilibrium 
points since the combined Poincaré index of both of them would be 0 (if the disease-free equilibrium point were a saddle point) or +2 (if the disease-free equilibrium point were not a saddle point). So, in any case, such a combined Poincaré index is not +1 compatible with the existence of some limit cycle surrounding both equilibrium points. As a result, the endemic equilibrium point is globally asymptotically stable if $R_{0}(q)>1$ (Theorem 3 ).

(c) If $R_{0}(q)=1$, then the disease-free and the endemic equilibrium points are coincident. From the non-negativity property of the solution for any given non-negative initial conditions (Theorem 1 (i)), it follows that a limit cycle surrounding the equilibrium point cannot exist in the phase plane (E, I). Also, by inspection of (1), it follows that $S(t)$ and $R(t)$ can only have periodic asymptotic solutions if $I(t)$ and $E(t)$ have asymptotic periodic solutions. As a result, the stability is also asymptotic and global in this case.

\section{SEIR Model with Impulsive Vaccination}

\subsection{The Epidemic Model Under Periodic Impulsive Vaccination}

Consider a modification of Equation (1) as follows with $q=0$ (no vaccination of newborns) and under a periodic impulsive vaccination of gain $p \in[0,1]$ and period $T$ at the time instants $t=n T$ for $n \in \mathbf{Z}_{0+}$ :

$$
\begin{gathered}
\dot{S}(t)=A-(\beta I+\mu) S(t)-p S\left(n T^{-}\right) \delta(t-n T) \\
\dot{E}(t)=\beta S(t) I(t)-(\mu+\sigma) E(t) \\
\dot{I}(t)=\sigma E(t)-(\mu+\gamma+\alpha) I(t) \\
\dot{R}=\gamma I-\mu R+p S\left(n T^{-}\right) \delta(t-n T)
\end{gathered}
$$

for all $t \in[n T,(n+1) T) ; \forall n \in \mathbf{Z}_{0+}$ with initial conditions $S(0) \geq 0, E(0) \geq 0, I(0) \geq 0$, and $R(0) \geq 0$ for $n=0$, where $\delta(t)$ is the Dirac distribution which reflects in the model dynamics the presence of a very intensive vaccination (ideally of impulsive infinity gain) concentrated in very short time intervals (ideally instantaneously administered at the time instants $t_{n}=n T$ ). The notation $S\left(n T^{-}\right.$) stands for the left limit of $S(t)$ at $t_{n}=n T$ while the right limit (i.e., the value as the impulse at $t_{n}$ is accounted for) is simply denoted as $S(n T)=S\left(n T^{+}\right)=(1-p) S\left(n T^{-}\right)$. The removed susceptible individuals at the impulsive time instants become recovered, which is reflected by the solution of the above fourth equation at the impulsive time instants, that is, $R(n T)=R\left(n T^{+}\right)=R\left(n T^{-}\right)+p S\left(n T^{-}\right)$. Note that $p=0$ describes the absence of impulsive vaccination while $p=1$ describes the total vaccination of the susceptible at the time instants $t_{n}=n T$, since no susceptible individual could be contacted by an infectious one because of the total removal of the first ones from the environment under study. It can be pointed out that impulsive vaccination is equivalent to intervention measures like isolation or confinement since a number of susceptible are transferred to the recovered subpopulation. The above-mentioned equivalence is at the level of transitions between subpopulations although no immunity is expected from such a state transition from susceptible to recovered. In this sense, the total recovered subpopulation is understood in a very wide sense as those individuals who are not susceptible to being infected, rather than just as those which are in fact immune, while those being immune are also included in the recovered subpopulation.

Note that Equation (3) is still valid for the dynamics of the total population. That means that there are no finite jumps of the total population at the impulsive time instants (in particular, $N_{*}=N_{*}^{-}=A / \mu$ at the disease-free equilibrium point) and there are no jumps either of the exposed or the infectious subpopulations at the disease-free equilibrium point.

By equalizing to zero the time-derivatives of the four subpopulations in Equation (21) while zeroing the exposed and infectious values, one gets the subsequent disease-free steady-state:

$$
S_{*}=\lim _{n \rightarrow \infty} S(n T)=\frac{A(1-p)\left(1-e^{-\mu T}\right)}{\mu\left(1-(1-p) e^{-\mu T}\right)} ; S_{*}^{-}=\lim _{n \rightarrow \infty} S\left(n T^{-}\right)=\frac{S_{*}}{1-p}=\frac{A\left(1-e^{-\mu T}\right)}{\mu\left(1-(1-p) e^{-\mu T}\right)}
$$




$$
R_{*}=\lim _{n \rightarrow \infty} R(n T)=N_{*}-S_{*}=\frac{A p}{\mu\left(1-(1-p) e^{-\mu T}\right)} ; R_{*}^{-}=\lim _{n \rightarrow \infty} R\left(n T^{-}\right)=N_{*}-S_{*}^{-}=\frac{A p e^{-\mu T}}{\mu\left(1-(1-p) e^{-\mu T}\right)}
$$

with $E_{*}=E_{*}^{-}=I_{*}=I_{*}^{-}=0$.

Remark 5. Note that if $q=p=0$ (i.e., in the absence of both newborns and impulsive vaccination efforts) then the disease-free equilibrium point discussed in the former section coincides with the above disease-free steady state. Note also that Equations (22) and (23) imply that the disease-free steady state is a periodic oscillation rather than an equilibrium point for nonzero $p$.

\subsection{Periodic Solutions and Stability Results}

The disease-free periodic solution and its local stability property are characterized in the following through the ad hoc use of Floquet theory. For this purpose, the following result is stated and proven.

Theorem 4. The following properties hold:

(i) Any solution of Equation (21) is non-negative for all time for any given set of non-negative initial conditions.

(ii) There exists a non-negative periodic disease-free solution of Equation (21) for each given set of non-negative finite initial conditions of the form

$$
\begin{gathered}
\hat{E}(t)=\hat{I}(t)=0 \\
\hat{S}(t)=\frac{A}{\mu}\left[1-\frac{p}{1-(1-p) e^{-\mu T}}\left(e^{-\mu\left(t-t_{0}\right)}+\left(1-e^{-\mu T}\right) \int_{t_{0}}^{t} \delta(\tau-n T) d \tau\right)\right] \\
=\frac{A}{\mu}\left[1-\left(1-\frac{(1-p)\left(1-e^{-\mu T}\right)}{1-(1-p) e^{-\mu T}}\right) e^{-\mu(t-(n-1) T)}\right] \\
\hat{R}(t)=\frac{A}{\mu} \frac{p}{1-(1-p) e^{-\mu T}}\left(e^{-\mu\left(t-t_{0}\right)}+\left(1-e^{-\mu T}\right) \int_{t_{0}}^{t} \delta(\tau-n T) d \tau\right) \\
=\frac{A}{\mu}\left(1-\frac{(1-p)\left(1-e^{-\mu T}\right)}{1-(1-p) e^{-\mu T}}\right) e^{-\mu(t-(n-1) T)} \\
; \forall t \in\left[t_{0}=(n-1) T, n T\right), \forall n \in \mathbf{Z}_{+}
\end{gathered}
$$

(iii) The above periodic disease-free solution converges asymptotically to finite non-negative left and right limits in Equations (22) and (23) with $E_{*}=E_{*}^{-}=I_{*}=I_{*}^{-}=0$ at the impulsive time instants $t_{n}=n T$ as $n \rightarrow \infty$.

(iv) The above periodic disease-free solution is locally asymptotically stable if the following constraint holds:

$$
T(\alpha+\sigma+\gamma+2 \mu)>\int_{0}^{T} \sqrt{(\alpha+\gamma-\sigma)^{2}+4 \beta \sigma \hat{S}(t)} d t ; \forall t \in\left[t_{0}=(n-1) T, n T\right), \forall n \in \mathbf{Z}_{+}
$$

(v) A sufficient condition for Property (iv) to hold is that the transmission rate be sufficiently small according to:

$$
\begin{gathered}
\beta \leq \beta_{c}(p, T)=\frac{\mu\left[1-(1-p) e^{-\mu T}\right]}{2 A \sigma\left(1-e^{-\mu T}\right)}\left[(\alpha+\sigma+\gamma+2 \mu)^{2}-(\alpha+\gamma-\sigma)^{2}\right] \\
=\frac{\mu\left[1-(1-p) e^{-\mu T}\right]}{2 A \sigma\left(1-e^{-\mu T}\right)}[2 \mu(\mu+\sigma)+(\alpha+\gamma)(2 \mu+3 \sigma)]
\end{gathered}
$$

Proof. From Theorem 1 with $q=0$, and since Equation (21) is subject to initial conditions $S(0) \geq 0$, $E(0) \geq 0, I(0) \geq 0$, and $R(0) \geq 0$, it follows that $S(t) \geq 0, E(t) \geq 0, I(t) \geq 0$, and $R(t) \geq 0 ; \forall t \in[0, T)$. One also has that $E(T)=E\left(T^{-}\right) \geq 0, I(T)=I\left(T^{-}\right) \geq 0, S(T)=(1-p) S\left(T^{-}\right) \geq 0$, and $R(T)=R\left(T^{-}\right)+p S\left(T^{-}\right) \geq 0$. So, one has $S(t) \geq 0, E(t) \geq 0, I(t) \geq 0$, and $R(t) \geq 0 ; \forall t \in[0, T)$. Then, it exists some $t_{k}=k T$ for $k \in \mathbf{Z}_{+}$(for some integer $k \geq 1$ ) such that $S(t) \geq 0, E(t) \geq 0, I(t) \geq 0$, and $R(t) \geq 0 ; \forall t \in[0, k T)$. Let $k_{0}=\left\{\max k \in \mathbf{Z}_{+}: \min (S(t), E(t), I(t), R(t)) \geq 0 ; \forall t \in[0, k T]\right\}$. Taking again initial conditions at $t_{0}=k_{0} T$, it follows that $S(t) \geq 0, E(t) \geq 0, I(t) \geq 0$, and $R(t) \geq 0 ; \forall t \in\left[0,\left(k_{0}+1\right) T\right]$. Furthermore, by complete induction, it follows that $S(t) \geq 0, E(t) \geq 0, I(t) \geq 0$, and $R(t) \geq 0 ; \forall t \in[0, k T)$ for any $k \in \mathbf{Z}_{0+}$. 
Then, any solution of Equation (21) for any given non-negative initial conditions is non-negative for all time. Property (i) has been proven. To prove Property (ii), first equalize to zero all the time-derivatives in Equation (21) to get $E(t)=I(t)=0$, and that the solution of the first differential equation is:

$$
S(t)=\frac{A}{\mu}\left(1-e^{-\mu(t-(n-1) T)}\right)+e^{-\mu(t-(n-1) T)} S[(n-1) T] ; \forall t \in[(n-1) T, n T), \forall n \in \mathbf{Z}_{+}
$$

In particular, the above equation leads to

$$
\begin{gathered}
S\left(n T^{-}\right)=e^{-\mu T} S[(n-1) T]+\frac{A}{\mu}\left(1-e^{-\mu T}\right) \\
S(n T)=(1-p) S\left(n T^{-}\right)=(1-p)\left(e^{-\mu T} S[(n-1) T]+\frac{A}{\mu}\left(1-e^{-\mu T}\right)\right)
\end{gathered}
$$

at the impulsive time instants. Denoting $S_{n}=S(n T)$ so that the stroboscopic mapping $F: S_{n-1} \rightarrow S_{n}$; $\forall n \in \mathbf{Z}_{+}$defined by

$$
S_{n}=F\left(S_{n-1}\right)=(1-p)\left(c+\left(S_{n-1}-c\right) e^{-\mu T}\right)
$$

with $c=A / \mu$, has a unique fixed point satisfying $S_{*}=F\left(S_{*}\right)$ defined by the first relationship of Equation (22). In this way, the delay-free periodic solution of the susceptible, whose right and left limit values at the impulsive time instants are the two equations of Equation (22) is:

$$
\hat{S}(t)=\frac{A}{\mu}\left[1-\left(1-\frac{(1-p)\left(1-e^{-\mu T}\right)}{1-(1-p) e^{-\mu T}}\right) e^{-\mu(t-(n-1) T)}\right] ; \forall t \in[(n-1) T, n T), \forall n \in \mathbf{Z}_{+}
$$

The periodic disease-free solution of the recovered is given by $\hat{R}(t)=\hat{N}(t)-\hat{S}(t)$, where $\hat{N}(t)$ is the periodic solution of the total population which satisfies under zero infection the differential equation:

$$
\dot{\hat{N}}(t)=A-\mu \hat{N}(t)
$$

whose transient solution is:

$$
\hat{N}(t)=\frac{A}{\mu}+\left(1-\frac{A}{\mu}\right) e^{-\mu t}
$$

Taking limits as $t \rightarrow \infty$ and subtracting Equation (31) from Equation (33) leads to the claimed steady-state periodic recovered oscillatory solution $\hat{S}(t)=\lim _{t \rightarrow \infty}(\hat{N}(t))-\hat{R}(t)=A / \mu-\hat{R}(t)$. Property (ii) has been proven. Property (iii) is a direct consequence of Equations (22) and (23) for the disease-free solution. To prove Property (iv), the periodic oscillation is firstly perturbed with a small perturbation $(s(t), e(t), i(t), r(t))$, that is,

$$
S(t)=\hat{S}(t)+s(t) ; E(t)=\hat{E}(t)+e(t) ; I(t)=\hat{I}(t)+i(t) ; R(t)=\hat{R}(t)+r(t)
$$

which results in the following modification of the differential system Equation (21) after neglecting the contribution to perturbation terms of orders higher than one:

$$
\begin{gathered}
\dot{s}(t)=-\mu s(t)-\beta \hat{S}(t) i(t)-p \sum_{n=0}^{\infty} s\left(n T^{-}\right) \delta(t-n T) ; \dot{e}(t)=\beta \hat{S}(t) i(t)-(\mu+\sigma) e(t) ; \\
\dot{i}(t)=\sigma e(t)-(\mu+\gamma+\alpha) i(t) ; \dot{r}(t)=\gamma i(t)-\mu r(t)+p \sum_{n=0}^{\infty} s\left(n T^{-}\right) \delta(t-n T)
\end{gathered}
$$

The periodic solution is stable if the perturbation vanishes through time. The function $\hat{S}(t)$ is given by Equation (31) and it is a periodic coefficient of period $T$ in Equation (35). The last equation is not needed for analysis if one considers the relationships $\hat{R}(t)=\hat{N}(t)-\hat{S}(t)=A / \mu-\hat{S}(t)$ for the disease-free solution. Therefore, a third-order differential system being built with the first three relations of Equation (35) might be compacted into a third-order differential system of the form 
$\dot{x}(t)=\mathbf{A}(t) x(t)$ with $\mathbf{A}(t)=\mathbf{A}(t+T)$ being a third-order periodic matrix function of dynamics for all $t \geq 0$. Its entries follow directly from inspection of Equation (35). Such a system has to be solved on a time interval $[0, T]$. A fundamental matrix $X(t)=X(t+T)$ of the third-order differential system, that is being nonsingular for all time since its columns are particular linearly independent solutions to initial conditions satisfying $X(0)=\mathbf{I}_{3}$, is defined for all $t=n T$ as $X(T)$, the monodromy matrix, being of the form:

$$
X(T)=\operatorname{Diag}\left((1-p) e^{-\mu T}, \quad \exp \left(-\frac{1}{2} \int_{0}^{T} P_{+}(t) d t\right), \quad \exp \left(-\frac{1}{2} \int_{0}^{T} P_{-}(t) d t\right)\right)
$$

where

$$
\begin{aligned}
& P_{+}(t)=\alpha+\sigma+\gamma+2 \mu+\sqrt{(\alpha+\gamma-\sigma)^{2}+4 \beta \sigma \hat{S}(t)} ; \forall t \in[0, T] \\
& P_{-}(t)=\alpha+\sigma+\gamma+2 \mu-\sqrt{(\alpha+\gamma-\sigma)^{2}+4 \beta \sigma \hat{S}(t)} ; \forall t \in[0, T] .
\end{aligned}
$$

The eigenvalues of the monodromy matrix, that is, its diagonal entries in this case are the Floquet multipliers [29,30], and the matrix $\mathbf{A}(t)$ is a stability matrix if the moduli of their Floquet multipliers are less than one. One gets by inspection of Equations (36)-(38) that the two first eigenvalues have a modulus less than one since $\mu>0,0 \leq p \leq 1$ and $P_{+}(t)>0 ; \forall t \in[0, T]$ and the third eigenvalue has a modulus less than one if and only if

$$
T(\alpha+\sigma+\gamma+2 \mu)>\int_{0}^{T} \sqrt{(\alpha+\gamma-\sigma)^{2}+4 \beta \sigma \hat{S}(t) d t}
$$

The condition Equation (39) implies that the periodic solution is locally asymptotically stable in the sense that the solution of the incremental system Equation (35) converges asymptotically to zero for sufficiently small initial conditions. Property (iv) has been proven. To prove Property (v), note that a sufficient condition for Equation (39) to hold is that

$$
(\alpha+\sigma+\gamma+2 \mu)^{2}>(\alpha+\gamma-\sigma)^{2}+4 \beta \sigma \max _{t \in[0, T]} \hat{S}(t)
$$

which holds if, equivalently, Equation (26) holds. Property (v) has been proven.

Remark 6. Theorem $4(v)$ gives a testable trade-off between the sizes of the critical transmission rate $\beta_{c}$ and those of the impulsive period ( $p$ and $T$ ) to guarantee the local stability of the periodic solution. In particular, for small periods for the impulsive vaccination actions, the critical transmission rate value compatible with the local asymptotic stability of the disease-free solution is high. That means that, in the main range of values of interest, the tolerance to the transmission rate guarantees that such a stability tolerance increases as the impulsive vaccination period decreases as intuitively expected. Note that it is not difficult to extend the performed study to the combination of the two combined vaccination strategies of vaccination of newborns and periodic impulsive vaccination. On the other hand, the generalization to impulsive vaccination controls under time-varying inter-impulse periods are an open design tool for related future work based on the adaptive sampling rate designs [43,44].

\section{Numerical Simulations}

The SEIR model is now evaluated under numerical simulations. The system of differential equations has been implemented in Python based on Odeint of the SciPy package which solves ordinary differential equations. The epidemiological parameters were taken from COVID-19 pandemic registered data in the background literature which result to be $\beta=0.57, \sigma=0.13, \gamma=0.067$ and $\alpha=0.01$ in day ${ }^{-1}[6-8]$. 


\subsection{Newborn Vaccination}

An initial total population of $N(0)=4 \times 10^{7}$ individuals with the following demographic parameters: $A=0.005$ (indivual $\times$ day) ${ }^{-1}$ and $\mu=0.005$ day $^{-1}$ was assumed. The model was evaluated under normalization to unity. The initial conditions of the subpopulations were $S(0)=1-1 / N(0)$, $E(0)=R(0)=0, I(0)=1 / N(0)$. The evolution in the absence of vaccination is displayed in Figure 2 . The basic reproduction number was $R_{0}=6.7$, and the solution converges to the endemic equilibrium point which is asymptotically stable with an infectious proportion of $I^{*}=0.05$. Figure 3 exhibits the evolution of the infectious for different levels of vaccination effort. Note that for $q>q_{c}=0.85$, the disease-free equilibrium point again becomes a stable attractor.

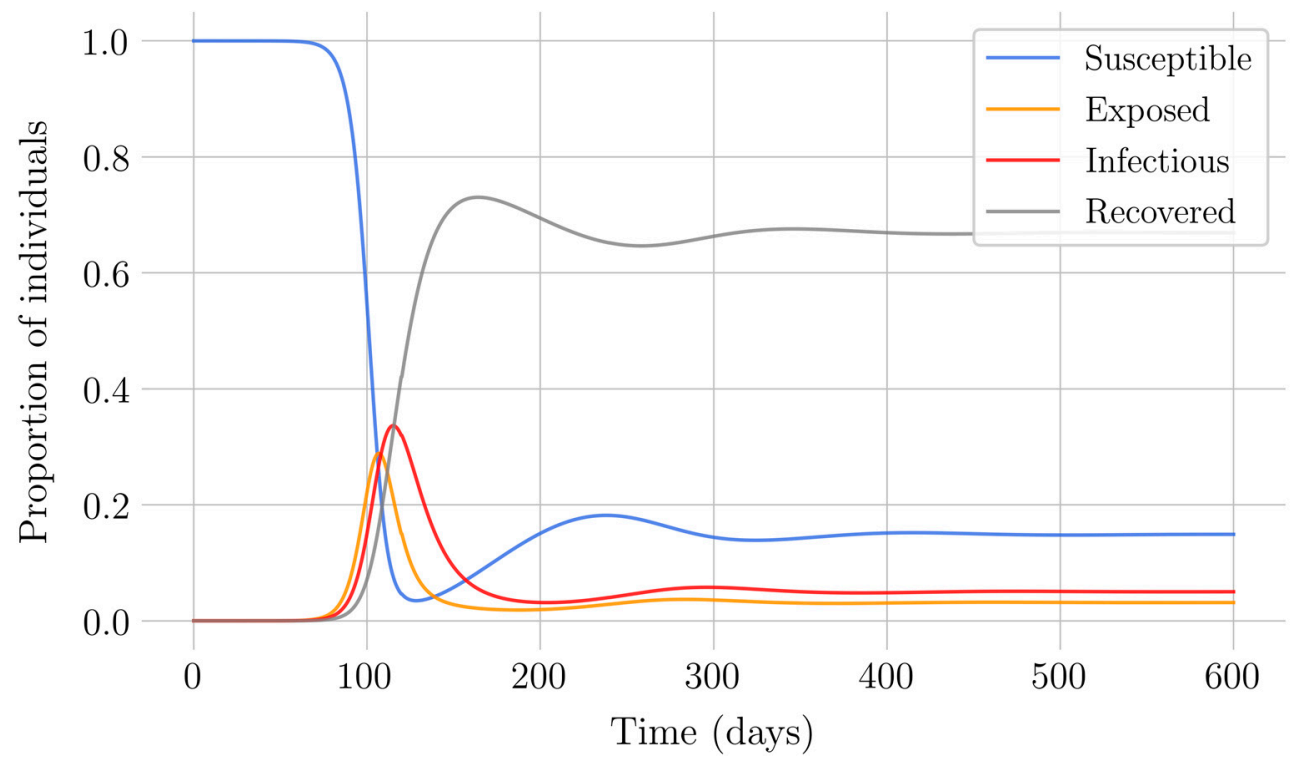

Figure 2. Evolution of the SEIR model in the absence of vaccination.

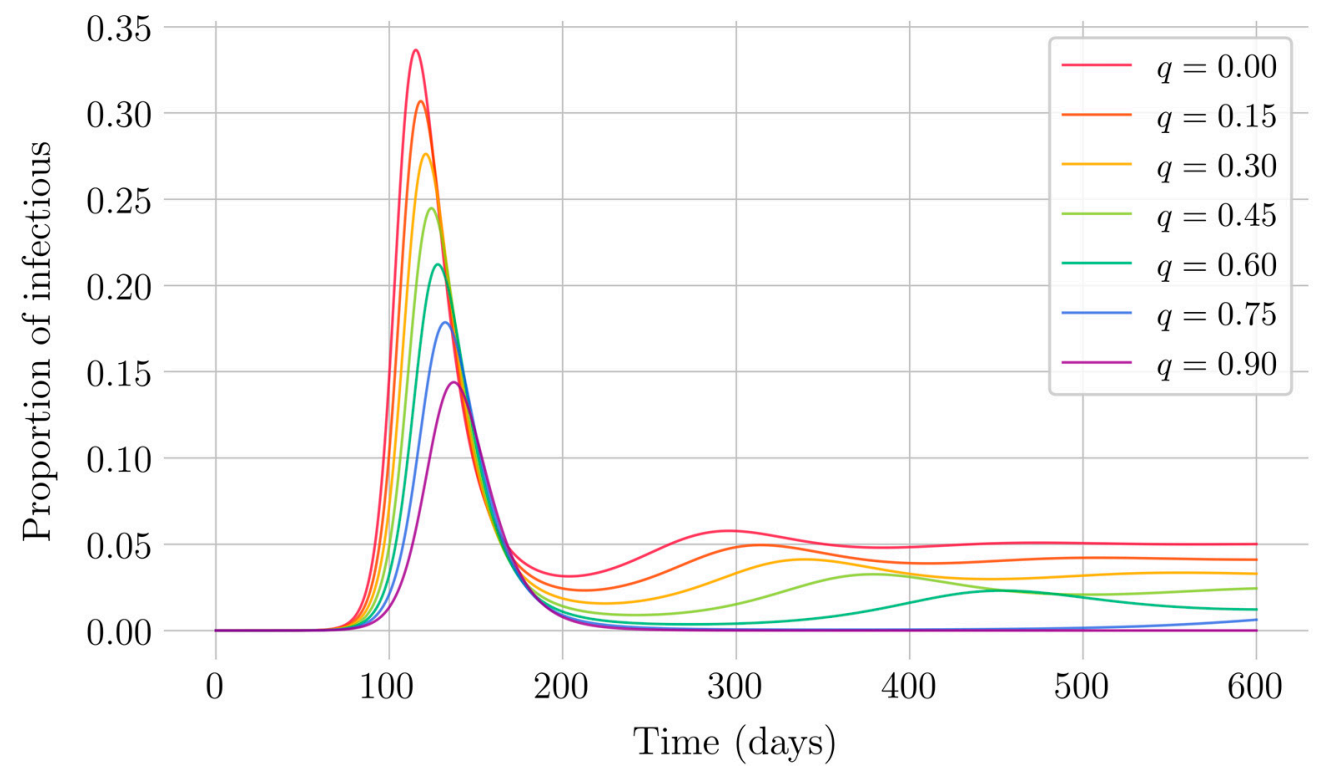

Figure 3. Evolution of the infectious proportion under different levels of vaccination effort.

It is now evaluated how to update the vaccination effort to the proportion of susceptible individuals through time. The basic idea is to increase the effort as the susceptible individuals increase. For that 
purpose, the following updating formula is used for the vaccination effort which adapts the vaccination effort to the proportion of susceptible through time:

$$
q(S(t))=\left(q_{\max }-q_{\min }\right) S(t)+q_{\min }
$$

Figures 4 and 5 show the model evolution with $q_{\max }=0.90$, and $q_{\min }=0.55$ and $q_{\min }=0.775$, respectively, under the newborns vaccination effort designed with the above formula. The endemic proportion of infectious is $I^{*}=0.015$ in the first case and $I^{*}=0.0036$ in the second one. Note that Figure 5 exhibits an improvement in the maximum infectious level at the expense of larger vaccination efforts according to the above formula.

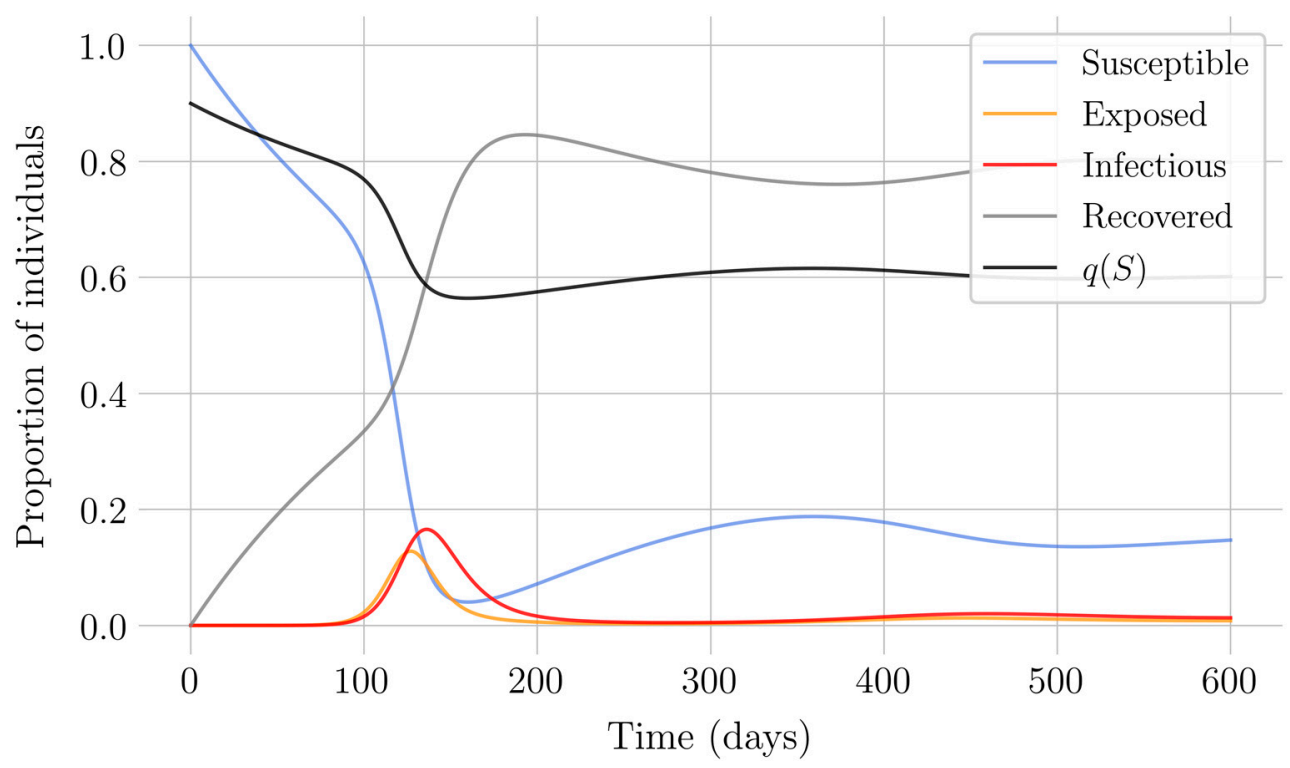

Figure 4. Evolution with $q_{\max }=0.90$ and $q_{\min }=0.55$.

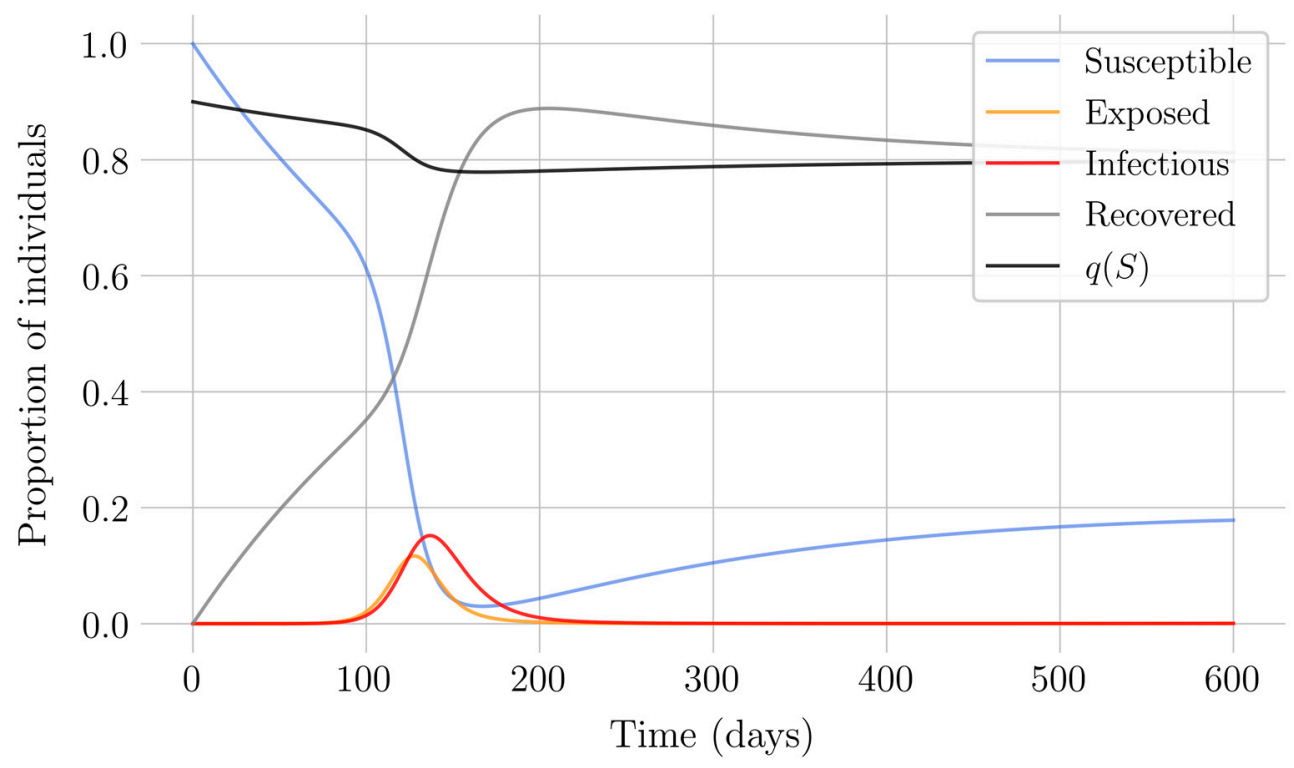

Figure 5. Evolution with $q_{\max }=0.90$ and $q_{\min }=0.775$. 


\subsection{Impulsive Vaccination}

Firstly, one considers an impulsive vaccination with parameters of vaccination proportion and impulse period $p=0.45$ and $T=120$ days. The evolution through time is displayed in Figure 6. It is seen that the evolution evolves to a periodic solution as expected.

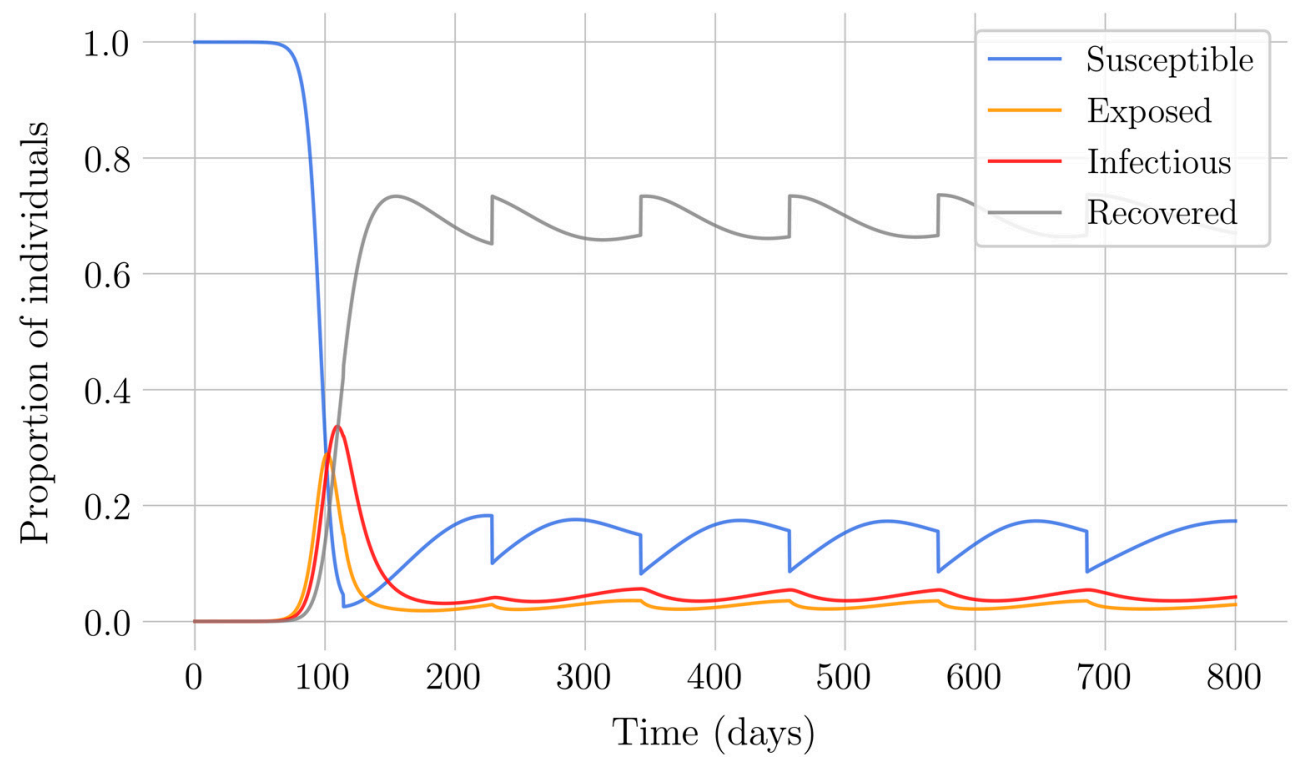

Figure 6. Evolution under impulsive vaccination with $p=0.45$ and $T=120$ days.

Figures 7 and 8 display the results for $T=120$ days as $p$ varies and $p=0.60$ as $T$ as varies, respectively. It can be seen that the results of the oscillation amplitude improve as $p$ increases and as $T$ decreases, as intuitively expected.

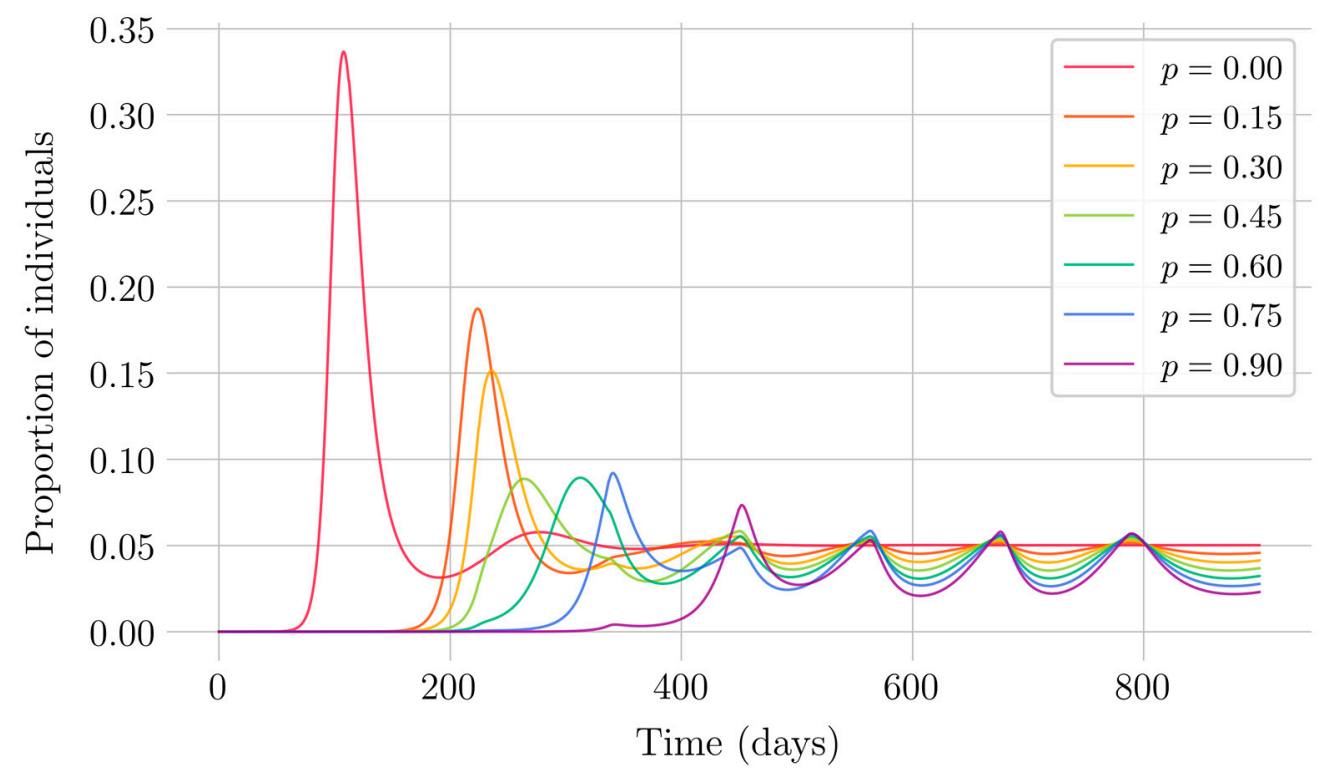

Figure 7. Infectious proportion evolution under impulsive vaccination for $T=120$ days. 


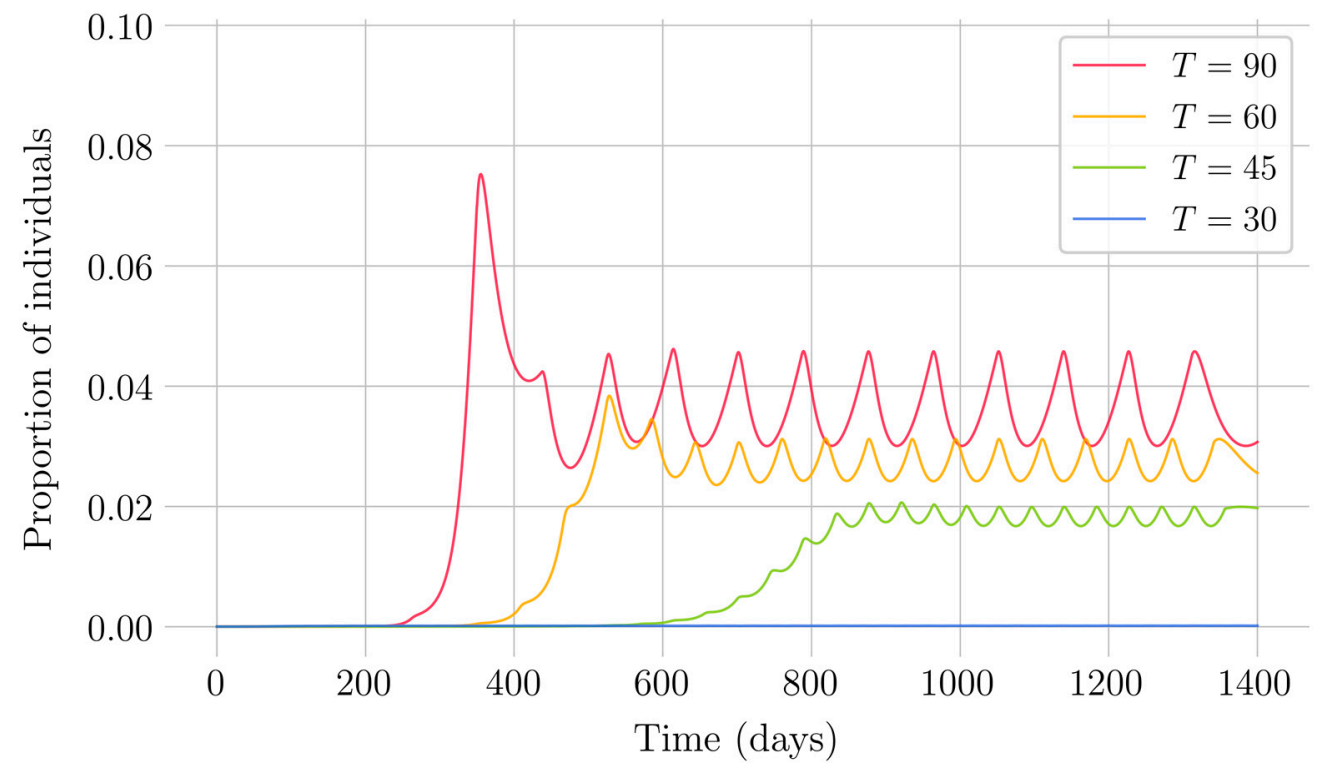

Figure 8. Infectious proportion evolution under impulsive vaccination for $p=0.60$.

The performance of increasing the vaccination effort as the susceptible individuals increase is now evaluated. For that purpose, the following updating formula is used for the vaccination effort which adapts the vaccination effort to the proportion of susceptible over time to improve the efficiency of the vaccination strategy:

$$
p(S(t))=\left(p_{\max }-p_{\min }\right) S(t)+p_{\min }
$$

Some tested results are shown in Figures 9 and 10 with $A=0.0045, p_{\max }=0.60, p_{\min }=0.30$, and $T=30$ days. The test is repeated in Figures 11 and 12 by increasing the minimum vaccination effort to $p_{\min }=0.55$ which improves the infection control and so improves the infection evolution, as expected.

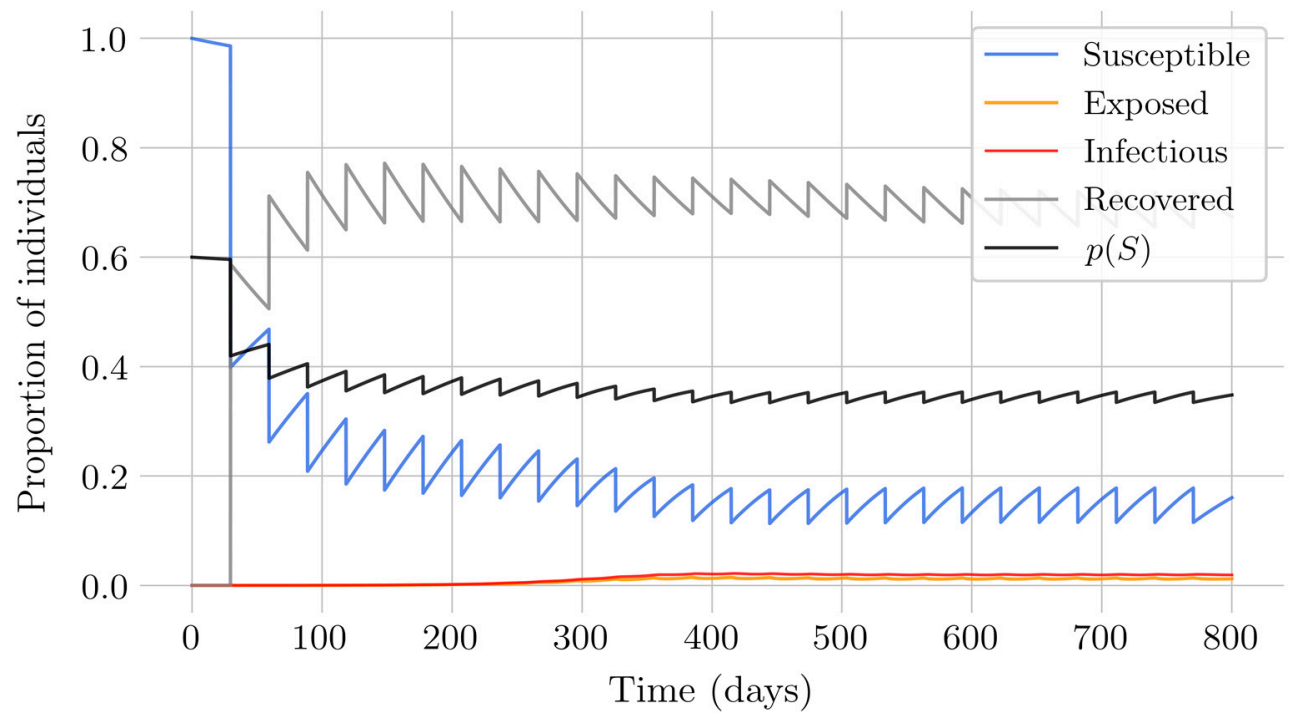

Figure 9. Model evolution for adapted impulsive vaccination with $A=0.0045, p_{\max }=0.60, p_{\min }=0.30$, $T=30$ days. 


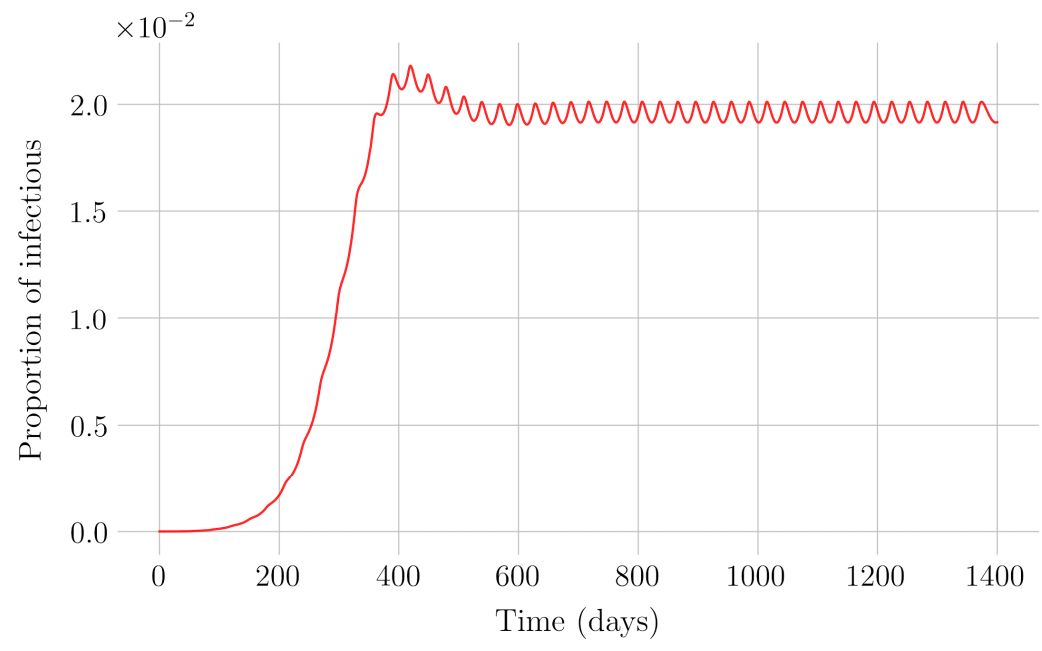

Figure 10. Detail of the infectious individuals proportion evolution for adapted impulsive vaccination with $A=0.0045, p_{\max }=0.60, p_{\min }=0.30, T=30$ days.

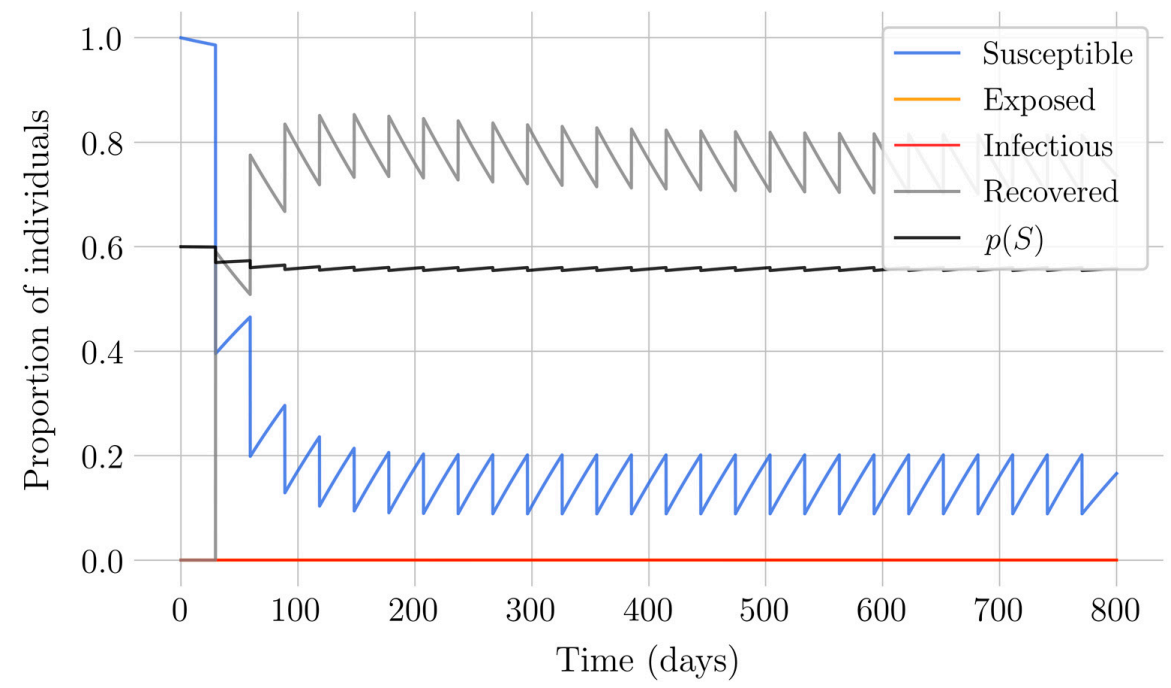

Figure 11. Model evolution for adapted impulsive vaccination with $A=0.0045, p_{\max }=0.60$, $p_{\min }=0.55, T=30$ days.

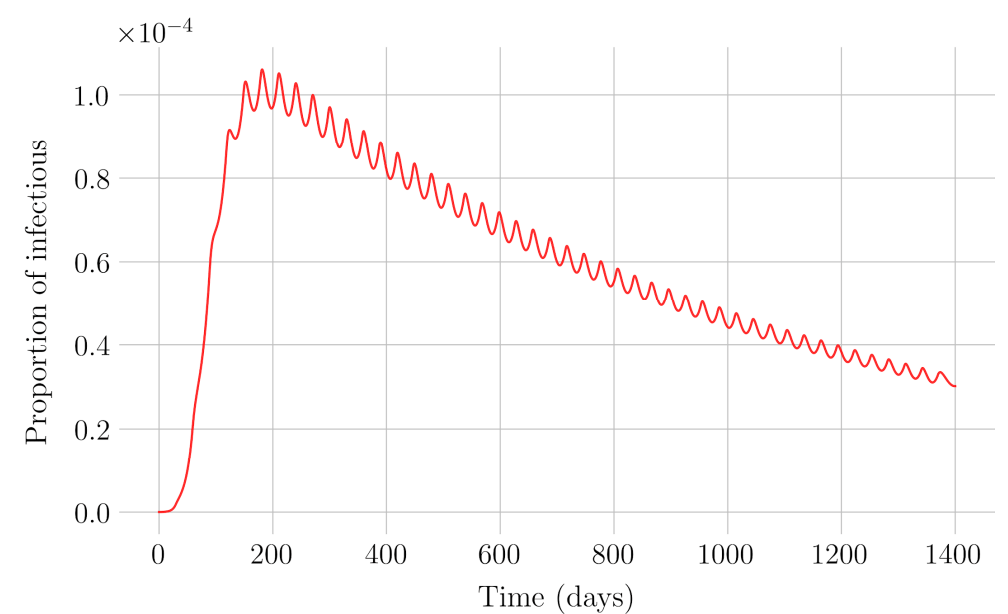

Figure 12. Detail of the infectious individuals proportion evolution for adapted impulsive vaccination with $A=0.0045, p_{\max }=0.60, p_{\min }=0.55, T=30$ days. 


\section{Conclusions}

In this work, the implementation of newborns and impulsive vaccination in the epidemiological model SEIR was studied. For this purpose, it was necessary to begin with an extensive theoretical framework in which the generalities of the model and the two vaccination strategies were explained, delving into some important concepts such as the existence and location of the equilibrium points or the state-steady periodic solutions and their stability properties, as well as their links to the ranges of values of the basic reproductive number. Although the central topic of the work is to study the influence of vaccination, it was considered important to devote a part of the paper body to these topics since feedback vaccination influences the allocation of the equilibrium states and the rates of convergence related to the absence of vaccination. In order to further study the vaccination model and control strategies, a part of the article has been devoted to stability analysis. In the case of newborn vaccination, the disease-free and endemic equilibrium points were found and Lyapunov's first method was applied to verify their local stability properties. It was proven that the endemic equilibrium point is not reachable as the disease-free one is locally asymptotically stable while the endemic one is reachable as the disease-free one is unstable. Those facts also lead to the conclusion that the respective local stability properties are also global since any solution is bounded for finite non-negative initial conditions.

In the case of impulsive vaccination, the calculations were more involved due to the impulsive nature of the system. It can be argued that the impulsive vaccination strategy may be of particular relevant interest in endemic situations when the disease exhibits successive decreasing and increasing levels of infection through time. Instead of equilibrium points, periodic solutions are found and characterized and, due to the complexity of the problem, only the disease-free periodic solution was obtained. To demonstrate the local stability of the calculated solution, Floquet's theory was applied, and in this way a stability condition containing an integral was obtained. Through some performed numerical simulations, it was possible to conclude, firstly, that both newborn and impulsive vaccination are effective strategies to eradicate the disease under sufficiently large levels of vaccination efforts. The required proportion of vaccination effort was seen to be relevant in both cases if the proportion of vaccines administered is proportional to the proportion of susceptible individuals. Secondly, it has been observed that the total coverage necessary to eradicate the disease was lower in the case of impulsive vaccination than in newborn vaccination, as mentioned in the theoretical framework. Regarding the strategy of impulsive vaccination, the local stability of a disease-free periodic solution was also demonstrated. Numerical simulations were developed by using background provided data for the disease parameterization of the SARS-COVID-19 pandemic. Although at this time there are no fully tested vaccines available for such a disease, the numerical examples adjusted to the formal setting indicate that the vaccination strategy improves the basic reproduction number and the disease is controlled more efficiently. It was also proven and tested through examples that the global vaccination strategy is better administrated if the vaccination effort is proportional to the susceptible subpopulation through time.

Author Contributions: Conceptualization, S.A.-Q.; data curation, M.E.-E.; formal analysis, M.E.-E. and S.A.-Q.; Investigation, M.E.-E. and S.A.-Q.; methodology and validation, S.A.-Q., M.E.-E. and M.D.1.S.; writing—original draft preparation, M.E.-E. and S.A.-Q.; software, M.E.-E.; resources, M.E.-E. and S.A.-Q.; supervision, S.A.-Q. and M.D.I.S.; visualization, M.E.-E.; writing-review and editing, M.D.I.S. and M.E.-E.; funding acquisition, M.D.1.S.; project administration, M.D.l.S. All authors have read and agreed to the published version of the manuscript.

Funding: This research was supported by the Spanish Institute of Health Carlos III through Grant COV 20/01213 and by the Spanish Government and European Commission through Grant RTI2018-094336-B-I00 (MCIU/AEI/FEDER, UE).

Acknowledgments: The authors are grateful to the Spanish Government and European Commission for Grant RTI2018-094336-B-I00 (MCIU/AEI/FEDER, UE) and to the Basque Government for Grant IT1207-19. They also thank the Spanish Institute of Health Carlos III for its support through Grant COV 20/01213. Finally, they thank the anonymous referees for their useful suggestions.

Conflicts of Interest: The authors declare that they have no competing interest regarding the publication of this manuscript. 


\section{References}

1. Keeling, M.J.; Rohani, P. Modelling Infectious Diseases; Princeton University Press: Princeton, NJ, USA, 2007.

2. Kermack, W.O.; McKendrick, A.G. A contribution to the mathematical theory of epidemics. Proc. R. Soc. Lond. Ser. A Math. Phys. Sci. 1927, 115, 700-721. [CrossRef]

3. Biswas, H.A.; Paiva, L.T.; De Pinho, M.D.R. A SEIR model for control of infectious diseases with constraints. Math. Biosci. Eng. 2014, 11, 761-784. [CrossRef]

4. Parsamanesh, M.; Erfanian, M. Global dynamics of an epidemic model with standard incidence rate and vaccination strategy. Chaos Solitons Fractals 2018, 117, 192-199. [CrossRef]

5. Bacaër, N. The model of Kermack and McKendrick for the plague epidemic in Bombay and the type reproduction number with seasonality. J. Math. Biol. 2012, 64, 403-422. [CrossRef] [PubMed]

6. Giordano, G.; Blanchini, F.; Bruno, R.; Colaneri, P.; Di Filippo, A.; Di Matteo, A.; Colaneri, M. Modelling the COVID-19 epidemic and implementation of population-wide interventions in Italy. Nat. Med. 2020, 26, 855-860. [CrossRef]

7. Yang, Z.; Zeng, Z.; Wang, K.; Wong, S.-S.; Liang, W.; Zanin, M.; Liu, P.; Cao, X.; Gao, Z.; Mai, Z.; et al. Modified SEIR and AI prediction of the epidemics trend of COVID-19 in China under public health interventions. J. Thorac. Dis. 2020, 12, 165-174. [CrossRef]

8. Yang, C.Y.; Wang, J. A mathematical model for the novel coronavirus epidemic in Wuhan, China. Math. Biosci. Eng. 2020, 17, 2708-2724. [CrossRef]

9. Nokes, D.J.; Swinton, J. Vaccination in pulses: A strategy for global eradication of measles and polio? Trends Microbiol. 1997, 5, 14-19. [CrossRef]

10. Shulgin, B. Pulse vaccination strategy in the SIR epidemic model. Bull. Math. Biol. 1998, 60, 1123-1148. [CrossRef]

11. Sisodiya, O.S.; Misra, O.P.; Dhar, J. Dynamics of cholera epidemics with impulsive vaccination and disinfection. Math. Biosci. 2018, 298, 46-57. [CrossRef]

12. Last, J.M. Dictionary of Epidemiology; Oxford University Press: New York, NY, USA, 2001.

13. Brauer, F.; Feng, Z.; Castillo-Chavez, C. Discrete epidemic models. Math. Biosci. Eng. 2010, 7, 1-15. [CrossRef] [PubMed]

14. Allen, L.J. A primer on stochastic epidemic models: Formulation, numerical simulation, and analysis. Infect. Dis. Model. 2017, 2, 128-142. [CrossRef] [PubMed]

15. Baxter, D. Active and passive immunity, vaccine types, excipients and licensing. Occup. Med. 2007, 57, 552-556. [CrossRef] [PubMed]

16. Heffernan, J.M.; Smith, R.J.; Wahl, L.M. Perspectives on the basic reproductive ratio. J. R. Soc. Interface 2005, 2, 281-293. [CrossRef]

17. Asociacioón Espanñola de Pediatría; Comité Asesor de Vacunas. Calendario de Vacunaciones de la Asociación Espanola de Pediatrńa: Recomendaciones. 2020. Available online: https://vacunasaep.org/profesionales/ calendario-de-vacunaciones-de-la-aep-2020 (accessed on 1 April 2020).

18. Organizacioón Mundial de la Salud. Cobertura Vacunal. 2020. Available online: https:/www.who.int/es/ news-room/fact-sheets/detail/immunization-coverage (accessed on 1 April 2020).

19. John, T.J.; Vashishtha, V.M. Eradicating poliomyelitis: India's journey from hyperendemic to polio-free status. Indian J. Med. Res. 2013, 137, 881-894.

20. Noticias ONU. La Falta de Pruebas Para Detectar el Coronavirus Oculta Casos y Muertes en América Latina. 2020. Available online: https://news.un.org/es/story/2020/04/1473512 (accessed on 15 April 2020).

21. Organización Mundial de la Salud. Rastreo de los Contactos en Situaciones de Brotes Epidémicos. 2017. Available online: https://www.who.int/features/qa/contact-tracing/es/ (accessed on 15 April 2020).

22. Zastrow, M. South Korea is reporting intimate details of COVID-19 cases: Has it helped? Nature 2020. [CrossRef]

23. Sun, K.; Viboud, C. Impact of contact tracing on SARS-CoV-2 transmission. Lancet Infect. Dis. 2020, 20, 876-877. [CrossRef]

24. Jonker, W. The European Struggle with COVID-19 Contact Tracing Apps. European Institute of Innovation \& Technology, 2020. Available online: https:/eit.europa.eu/news-events/news/european-struggle-covid-19contact-tracing-apps (accessed on 15 April 2020). 
25. Transparencia Sobre el Nuevo Coronavirus (COVID-19). euskadi.eus. 2020. Available online: https://www. euskadi.eus/preguntas-sobre-el-confinamiento-cuarentena/web01-a3korona/es/ (accessed on 15 April 2020).

26. De La Sen, M.; Nistal, R.; Alonso-Quesada, S.; Ibeas, A. Some Formal Results on Positivity, Stability, and Endemic Steady-State Attainability Based on Linear Algebraic Tools for a Class of Epidemic Models with Eventual Incommensurate Delays. Discret. Dyn. Nat. Soc. 2019, 2019, 8959681. [CrossRef]

27. Strogatz, S.H. Nonlinear Dynamics and Chaos; Perseus Books: Reading, UK, 1994.

28. Chen, G. Stability of Nonlinear Systems. In Encyclopedia of RF and Microwave Engineering; Chang, K., Ed.; John Wiley \& Sons: Hoboken, NJ, USA, 2005; pp. 4881-4896.

29. Klausmeier, C.A. Floquet theory: A useful tool for understanding nonequilibrium dynamics. Theor. Ecol. 2008, 1, 153-161. [CrossRef]

30. Bittani, S.; Colaneri, P. Periodic Systems: Filtering and Control; Springer: London, UK, 2009. [CrossRef]

31. DeJesus, E.X.; Kaufman, C. Routh-Hurwitz criterion in the examination of eigenvalues of a system of nonlinear ordinary differential equations. Phys. Rev. A 1987, 35, 5288-5290. [CrossRef]

32. Khan, M.A.; Ullah, S.; Ullah, S.; Farhan, M. Fractional order SEIR model with generalized incidence rate. AIMS Math. 2020, 5, 2843-2857. [CrossRef]

33. He, S.; Peng, Y.; Sun, K. SEIR modeling of the COVID-19 and its dynamics. Nonlinear Dyn. 2020, 101, 1667-1680. [CrossRef] [PubMed]

34. Pandey, G.; Chaudhary, P.; Gupta, R.; Pal, S. SEIR and regression model based COVID-19 outbreak predictions in India. arXiv 2020, arXiv:2004.00958.

35. Annas, S.; Pratama, M.I.; Rifandi, M.; Sanusi, W.; Side, S. Stability analysis and numerical simulation of SEIR model for pandemic COVID-19 spread in Indonesia. Chaos Solitons Fractals 2020, 139, 110072. [CrossRef] [PubMed]

36. Fabiano, N.; Radenović, S. On COVID-19 diffusion in Italy: Data analysis and possible outcome. Vojn. Glas. 2020, 68, 216-224. [CrossRef]

37. Wang, W.; Wu, Z.-N.; Wang, C.; Hu, R. Modelling the spreading rate of controlled communicable epidemics through an entropy-based thermodynamic model. Sci. China Ser. G Phys. Mech. Astron. 2013, 56, 2143-2150. [CrossRef]

38. De La Sen, M.; Nistal, R.; Ibeas, A.; Garrido, A.J. On the Use of Entropy Issues to Evaluate and Control the Transients in Some Epidemic Models. Entropy 2020, 22, 534. [CrossRef]

39. De La Sen, M.; Agarwal, R.P.; Ibeas, A.; Alonso-Quesada, S. On a Generalized Time-Varying SEIR Epidemic Model with Mixed Point and Distributed Time-Varying Delays and Combined Regular and Impulsive Vaccination Controls. Adv. Differ. Equ. 2010, 2010, 281612. [CrossRef]

40. Beretta, E.; Kolmanovskii, V.; Shaikhet, L. Stability of epidemic model with time delays influenced by stochastic perturbations. Math. Comput. Simul. 1998, 45, 269-277. [CrossRef]

41. Santonja, F.; Shaikhet, L. Probabilistic stability analysis of social obesity epidemic by a delayed stochastic model. Nonlinear Anal. Real. World Appl. 2014, 17, 114-125. [CrossRef]

42. D'Onofrio, A. Stability properties of pulse vaccination strategy in SEIR epidemic model. Math. Biosci. 2002, 179, 57-72. [CrossRef]

43. De La Sen, M. Adaptive sampling for improving the adaptation transients in hybrid adaptive control. Int. J. Control 1985, 41, 1189-1205. [CrossRef]

44. Delasen, M. A method for improving the adaptation transient using adaptive sampling. Int. J. Control 1984, 40, 639-665.

45. De Jesus, L.F.; Silva, C.M.; Vilarinho, H. Periodic orbits for periodic eco-epidemiological systems with infected prey. Electron. J. Qual. Theory Differ. Equ. 2020, 54, 1-20. [CrossRef]

Publisher's Note: MDPI stays neutral with regard to jurisdictional claims in published maps and institutional affiliations. 